\title{
Geometric pressure in real and complex 1-dimensional dynamics via trees of pre-images and via spanning sets
}

\author{
Feliks Przytycki ${ }^{1}$
}

Received: 27 February 2016 / Accepted: 5 November 2017 / Published online: 21 November 2017 C The Author(s) 2017. This article is an open access publication

\begin{abstract}
We consider $f: \widehat{I} \rightarrow \mathbb{R}$ being a $C^{3}$ (or $C^{2}$ with bounded distortion) realvalued multimodal map with non-flat critical points, defined on $\widehat{I}$ being the union of closed intervals, and its restriction to the maximal forward invariant subset $K \subset \widehat{I}$. We assume that $\left.f\right|_{K}$ is topologically transitive and, usually, of positive topological entropy. We call this setting the generalized real multimodal case. We consider also $f: \widehat{\mathbb{C}} \rightarrow \widehat{\mathbb{C}}$ a rational map on the Riemann sphere and its restriction to $K=J(f)$ being Julia set, the complex case. We consider topological pressure $P_{\text {spanning }}(t)$ for the potential function $\varphi_{t}=-t \log \left|f^{\prime}\right|$ for $t>0$ and iteration of $f$ defined in a standard way using $(n, \varepsilon)$-spanning sets. Despite of $\phi_{t}=\infty$ at critical points of $f$, this definition makes sense (unlike the standard definition using $(n, \varepsilon)$-separated sets) and we prove that $P_{\text {spanning }}(t)$ is equal to other pressure quantities, called for this potential geometric pressure, in the real case under mild additional assumptions, and in the complex case provided there is at most one critical point with forward trajectory accumulating in $J(f)$. $P_{\text {spanning }}(t)$ is proved to be finite for general rational maps, but it may occur infinite in the real case. We also prove that geometric tree pressure in the real case is the same for trees rooted at all safe points, in particular at all points except the set of Hausdorff dimension 0, the fact missing in Przytycki and Rivera-Letelier (Geometric pressure for multimodal maps of the interval, arXiv:1405.2443) proved in the complex case in Przytycki (Trans Am Math Soc 351:2081-2099, 1999).
\end{abstract}

Communicated by A. Constantin.

Feliks Przytycki: Partially supported by Polish NCN Grant "Chaos, fractals and conformal dynamics, III", 2014/13/B/ST1/01033.

Feliks Przytycki

feliksp@impan.pl

1 Institute of Mathematics, Polish Academy of Sciences, ul. Śniadeckich 8, 00-656 Warsaw, Poland 
Keywords 1-Dimensional dynamics - Geometric pressure - Spanning sets · Thermodynamical formalism

Mathematics Subject Classification Primary 37D35 ; Secondary 37E05 · 37F10

\section{Contents}

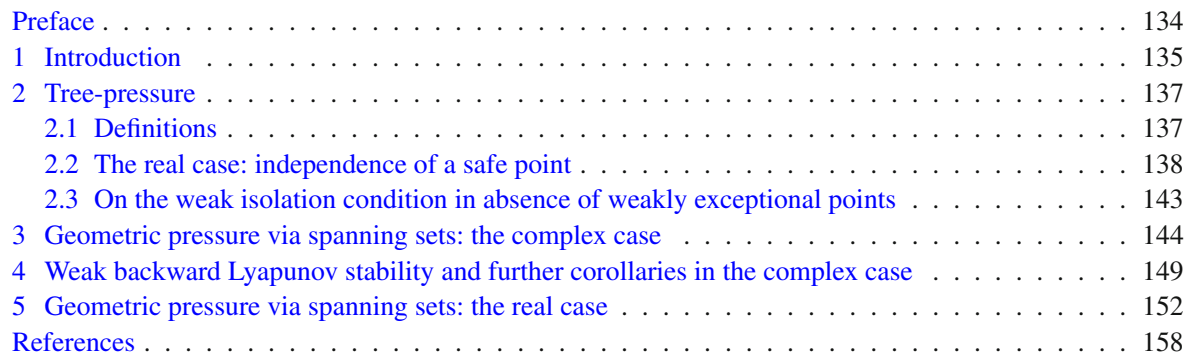

\section{Preface}

I dedicate this paper to the memory of Marian Smoluchowski ${ }^{1}$, one of founders of Statistical Physics, on 100 anniversary of his premature death.

In Markov chain models in Statistical Physics, the spaces of configurations of states (symbols, spins) over lattices, e.g. over $\mathbb{Z}^{d}$, see e.g. [21] or [22], are considered. One can restrict considerations to $\mathbb{Z}$, where the configurations are just sets of trajectories under an action of a function $f$ on a state space. Free energy is replaced by so-called topological pressure $P(\phi)=P(f, \phi)$, depending on a potential function $\phi$, replacing Hamiltonian. Equilibrium measures are considered, for which measure entropy + integral of potential attains the pressure.

In this paper, which is a complement to [18] and [16], we just study the pressure itself, various definitions and relations between them, in the case $\phi=-t \log \left|f^{\prime}\right|$ in the one-dimensional settings, real or complex. The difficulties are caused by the singularities of the derivative $f^{\prime}$ at critical points of $f$. As usual $t$ is a parameter, inverse of temperature. We call the pressure geometric since the potential $f^{\prime}$ is related to local geometry, in particular for many (so-called: hyperbolic) points $x$ we have $\left|\left(f^{n}\right)^{\prime}(x)\right|^{-1} \approx \operatorname{diam} B_{n}(x)$, where $B_{n}(x)$ is the connected component of the set $f^{-n}\left(B\left(f^{n}(x), \Delta\right)\right)$ containing $x$, for a constant $\Delta>0$. It corresponds to an $n$-th cylinder (a configuration fixed over $n+1$ consecutive integers) for a Markov (partition) model.

\footnotetext{
1 Marian Smoluchowski, 1872-1917, spent his young years in Vienna and graduated in physics at the University of Vienna; later he worked at Jan Kazimierz University in Lvov and Jagiellonian University in Cracow.
} 


\section{Introduction}

Let us start with the classical

Definition 1.1 (Topological pressure via separated sets) Let $f: X \rightarrow X$ be a continuous map of a compact metric space $(X, \rho)$ and $\phi: X \rightarrow \mathbb{R}$ be a real continuous function. For every positive integer $n$ and $x \in X$ denote $S_{n} \phi(x)=\sum_{j=0}^{n-1} \phi\left(f^{j}(x)\right)$. For every integer $n \geq 0$ define the metric $\rho_{n}(x, y)=\max \left\{\rho\left(f^{j}(x), f^{j}(y)\right): j=\right.$ $0, \ldots, n\}$. For every $\varepsilon>0$ a set $Y \subset X$ is said to be $(n, \varepsilon)$-separated if for every $y_{1}, y_{2} \in Y$ such that $y_{1} \neq y_{2}$ it holds that $\rho_{n}\left(y_{1}, y_{2}\right) \geq \varepsilon$. Define

$$
P_{\text {sep }}(f, \phi, \varepsilon):=\limsup _{n \rightarrow \infty} \frac{1}{n} \log \left(\sup _{Y} \sum_{y \in Y} \exp S_{n} \phi(y)\right),
$$

supremum taken over all $(n, \varepsilon)$-separated sets $Y \subset X$, and

$$
P_{\text {sep }}(f, \phi)=\lim _{\varepsilon \rightarrow 0} P_{\text {sep }}(f, \phi, \varepsilon)
$$

Analogously

$$
P_{\text {spanning }}(f, \phi, \varepsilon):=\limsup _{n \rightarrow \infty} \frac{1}{n} \log \left(\inf _{Y} \sum_{y \in Y} \exp S_{n} \phi(y)\right),
$$

infimum taken over all $(n, \varepsilon)$-spanning sets $Y \subset X$, i.e. such that for every $x \in X$ there exists $y \in Y$ such that $\rho_{n}(x, y)<\varepsilon$, in other words such that $\bigcup_{y \in Y} B_{\rho_{n}}(y, \varepsilon)=X$, and

$$
P_{\text {spanning }}(f, \phi):=\lim _{\varepsilon \rightarrow 0} P_{\text {spanning }}(f, \phi, \varepsilon) \text {. }
$$

It is easy to prove

Theorem 1.2 (see e.g. [24]) For every continuous $\phi: X \rightarrow \mathbb{R}$

$$
P_{\text {spanning }}(f, \phi)=P_{\text {sep }}(f, \phi) \text {. }
$$

This pressure depends on topology, but does not depend on metric.

This equality follows from

$$
P_{\text {sep }}(f, \phi, 2 \varepsilon) \leq P_{\text {spanning }}(f, \phi, \varepsilon) \leq P_{\text {sep }}(f, \phi, \varepsilon) .
$$

In this paper we shall discuss $\phi=\phi_{t}=-t \log \left|f^{\prime}\right|$ for all parameters $t>0$. This is slightly different from the previous situation in dimension 1 if $f$ is differentiable and has critical points, i.e. points $c \in X$ where the derivative of $f$ is 0 . At these points 
$\phi$ is not continuous. We assign to $\phi$ the value $+\infty$ there. The notion $P_{\text {sep }}$ does not make much sense in this case, as this quantity is equal to $+\infty$, by taking $Y$ containing some critical points, so it is replaced by the notion of tree pressure, see (2.1). However $P_{\text {spanning defined as above happens to make sense and a part of this paper is devoted }}$ to explaining this.

We discuss two settings:

1. (Complex) $f$ is a rational mapping of degree at least 2 of the Riemann sphere $\widehat{\mathbb{C}}$ usually with the spherical metric. We consider $f$ on its Julia set $K=J(f)$.

2. (Real) $f$ is a real generalized multimodal map. Namely it is defined on a neighbourhood $\mathbf{U} \subset \mathbb{R}$ of its compact invariant subset $K$. We assume $f \in C^{2}$, is non-flat at all its turning and inflection critical points, has bounded distortion property for its iterates (see the definition below), $\left.f\right|_{K}$ is topologically transitive (that is for every $U, V$ open in $K$ there exists $n>0$ such that $\left.f^{n}(U) \cap V \neq \emptyset\right)$ and has positive topological entropy on $K$.

Definition 1.3 (bounded distortion) Following [10] we say that for $\varepsilon>0$ and an interval $I \subset \mathbb{R}$, an interval $I^{\prime} \supset I$ is an $\varepsilon$-scaled neighbourhood of $I$ if $I^{\prime} \backslash I$ has two components, call them left and right, $L$ and $R$, such that $|L| /|I|,|R| /|I|=\varepsilon$.

Following [16, Definition 1.12] we say that a $C^{1}$ map $f: U \rightarrow \mathbb{R}$ for $U$ an open subset of $\mathbb{R}$ satisfies bounded distortion for its iterates, abbr. $\mathrm{BD}$, condition if there exists $\delta>0$ such that for every $\varepsilon>0$ there exists $C=C(\varepsilon)>0$ such that the following is true: for every pair of intervals $I_{1} \subset U, I_{2} \subset \mathbb{R}$ such that $\left|I_{2}\right| \leq \delta$ and for every $n>0$, if $f^{n}$ maps diffeomorphically an interval $I_{1}^{\prime}$ containing $I_{1}$ onto an interval $I_{2}^{\prime}$ being an $\varepsilon$-scaled neighbourhood of $I_{2}$ and $f^{n}\left(I_{1}\right)=I_{2}$, then for every $x, y \in I_{2}$ we have for $g=\left(\left.f^{n}\right|_{I_{1}}\right)^{-1}$,

$$
\left|g^{\prime}(x) / g^{\prime}(y)\right| \leq C(\varepsilon) .
$$

We assume also that $K$ is a maximal invariant subset on a finite union of pairwise disjoint closed intervals $\widehat{I}=\bigcup_{j} \widehat{I}_{j} \subset \mathbf{U}$ whose ends are in $K$. (This maximality corresponds to Darboux property, [16]).

We call a periodic orbit $x, f(x), \ldots f^{m}(x)=x$ (or just the periodic point $x$ ) hyperbolic repelling if $\left|\left(f^{m}\right)^{\prime}(x)\right|>1$, hyperbolic attracting if $\left|\left(f^{m}\right)^{\prime}(x)\right|>1$ and neutral if $\left|\left(f^{m}\right)^{\prime}(x)\right|=1,[10$, Ch.II.6]. By adjusting $\widehat{I}$ and $\mathbf{U}$ we can assume there are no critical points outside $K$, no attracting periodic orbits in $\mathbf{U}$ and no neutral periodic orbits in $\mathbf{U} \backslash K$.

If all the conditions above hold, we write $(f, K) \in \mathscr{A}_{+}^{\mathrm{BD}}$. The subscript + is to mark positive topological entropy. Sometimes we write $(f, K, \widehat{I}, \mathbf{U})$.

In place of BD one sometimes assumes just $C^{3}$ and denotes the related class by $\mathscr{A}_{+}^{3}$; together with other assumptions it leads to BD anyhow. E.g. if $(f, K) \in \mathscr{A}_{+}^{3}$ and all periodic orbits in $K$ are hyperbolic repelling, then, after an appropriate modification of $f$ outside a neighbourhood of $K$ if necessary, BD holds, see [16, Remark 2.14 and Lemma A.4].

If neither BD nor $C^{3}$ is assumed, we write $(f, K) \in \mathscr{A}_{+}$. If positive topological entropy is not assumed either, we just write $\mathscr{A}$. For a more detailed description of 
the real setting see [16]. Examples: sets in the spectral decomposition, except attracting periodic orbits and solenoidal attractors, see [10, Theorem III.4.2]. See also our Example 5.3.

In both settings the set of all critical points will be denoted by $\operatorname{Crit}(f)$.

The function $\phi_{t}$ is sometimes called the geometric potential and the pressure is called the geometric pressure, see e.g. [19].

This name is justified by

$$
\exp S_{n} \phi_{t}(z)=\left|\left(f^{n}\right)^{\prime}(z)\right|^{-t} \approx\left(\operatorname{diam} B_{n}(z)\right)^{t},
$$

where $B_{n}(z):=\operatorname{Comp}_{z}\left(f^{-n}\left(B\left(f^{n}(z), \Delta\right)\right)\right)$ for a constant $\Delta>0$ and an arbitrary $t>0$. Here $\operatorname{Comp}_{z}$ means the component in $\mathbb{C}$ or $\mathbb{R}$ containing $z$.

There exists often a unique equilibrium $f$-invariant measure $\mu_{t}$ on $K$ such that $\mu_{t}\left(B_{n}(z)\right) \approx \exp \left(S_{n} \phi_{t}(z)\right) \exp \left(-n P\left(\phi_{t}\right)\right)$. See e.g. [1, Theorem 1.22], [21], [15, Main Theorem], [16, Theorem A].

There are several equivalent definitions of geometric pressure $P\left(\phi_{t}\right)$, see $[18,19]$ or [16] in the interval case. One of them useful in this paper is

Definition 1.4 (hyperbolic pressure)

$$
P_{\text {hyp }}\left(f, \phi_{t}\right):=\sup _{X} P\left(\left.f\right|_{X},-\left.\phi_{t}\right|_{X}\right)
$$

supremum taken over all compact $f$-invariant (that is $f(X) \subset X$ ) isolated (Cantor) uniformly hyperbolic subsets of $K$. Sometimes we write $P_{\text {hyp }}(t)$.

Isolated (or forward locally maximal), means that there is a neighbourhood $U$ of $X$ such that $f^{n}(x) \in U$ for all $n \geq 0$ implies $x \in X$.

A set $X$ is said to be hyperbolic, uniformly hyperbolic or expanding if there is a constant $\lambda_{X}>1$ such that for all $n$ large enough and all $x \in X$ we have $\left|\left(f^{n}\right)^{\prime}(x)\right| \geq$ $\lambda_{X}^{n}$

\section{Tree-pressure}

\subsection{Definitions}

We devote this section to studying a modified definition of pressure by separated sets, useful when the one given by (1.1) does not make sense, called tree-pressure, see e.g. [19].

In the real and complex settings we define for all $t \in \mathbb{R}$

$$
\begin{aligned}
P_{\text {tree }}(z, t): & =\limsup _{n \rightarrow \infty} \frac{1}{n} \log Q_{n}(z, t), \\
\text { where } Q_{n}(z, t): & =\sum_{y \in f^{-n}(z) \cap K}\left|\left(f^{n}\right)^{\prime}(y)\right|^{-t} .
\end{aligned}
$$


Definition 2.1 A point $z \in K$ is said to be safe, or Crit $(f)$-safe if for every $\xi>0$ and all $n \geq n(\xi)>0$ large enough

$$
B(z, \exp (-\xi n)) \cap \bigcup_{j=1}^{n} f^{j}(\operatorname{Crit}(f))=\emptyset .
$$

It immediately follows from the definitions that Hausdorff dimension of the set of points which are not safe is equal to 0 .

In the complex setting the following is true:

Theorem 2.2 ([14,18]) For every rational $f: \widehat{\mathbb{C}} \rightarrow \widehat{\mathbb{C}}$ of degree at least 2 and for every $z \in K=J(f)$ safe and $t \in \mathbb{R}$

$$
P_{\text {tree }}(z, t)=P_{\text {hyp }}\left(f, \phi_{t}\right) \text {. }
$$

In particular in the complex case $P_{\text {tree }}(z, t)$ does not depend on $z$ safe; it is constant except $z$ in a set of Hausdorff dimension 0 . We denote this tree-pressure for $z$ safe by $P_{\text {tree }}(t)$.

\subsection{The real case: independence of a safe point}

In the generalized multimodal setting the above equality was known only for $z$ being safe, safe forward (in case $K$ is not weakly isolated) and hyperbolic, see [16, Lemma 4.4]. We recall the definitions mentioned here, compare [16]:

Definition 2.3 (hyperbolic) A point $z \in K$ is called hyperbolic (or expanding) if there exist $\lambda>1$ and $\Delta>0$ such that for all $n>0\left|\left(f^{n}\right)^{\prime}(z)\right| \geq$ Const $\lambda^{n}$ and $f^{n}$ maps diffeomorphically $\operatorname{Comp}_{z}\left(f^{-n}\left(B\left(f^{n}(z), \Delta\right)\right)\right)$ onto $B\left(f^{n}(z), \Delta\right)$.

Definition 2.4 (safe forward) A point $z \in K$ is called safe forward if there exists $\Delta>0$ such that $\operatorname{dist}\left(f^{j}(z), \partial \widehat{I}\right) \geq \Delta$ for all $j=0,1, \ldots$

Definition 2.5 (weak isolation) A compact set $K \subset \mathbb{R}$ is said to be weakly isolated for a continuous mapping on a neighbourhood of $K$ to $\mathbb{R}$ for which $K$ is forward invariant, if there exists $\varepsilon>0$ such that every $f$-periodic orbit $O(p) \subset B(K, \varepsilon)$ must be in $K$.

Though the set of all hyperbolic points has Hausdorff dimension equal to the hyperbolic dimension of $K$, i.e. supremum of Hausdorff dimensions of isolated uniformly hyperbolic subsets of $K$, which is the first zero of the hyperbolic pressure ([16, Proposition 1.21$]$ ), see the definition above, the complementary set can seemingly also be large. ${ }^{2}$

One of aims of this paper is to prove

\footnotetext{
2 I do not know any precise results on this, neither any reference. Note that the smaller set: of non-conical points with upper Lyapunov exponent positive, has Hausdorff dimension 0, see [4, Proposition 3.21].
} 
Theorem 2.6 For $(f, K) \in \mathscr{A}_{+}^{\mathrm{BD}}$, or for $(f, K) \in \mathscr{A}_{+}^{3}$, with all periodic orbits in $K$ hyperbolic repelling, if $K$ is weakly isolated and $t>0$, the tree pressure $P_{\text {tree }}(z, t)$ does not depend on $z \in K$ safe. In particular

$$
P_{\text {tree }}(z, t)=P_{\text {hyp }}\left(f, \phi_{t}\right) \text {. }
$$

Moreover limsup can be replaced by lim in the definition of tree pressure, i.e the limit exists.

As in the complex case we denote this tree-pressure for safe points by $P_{\text {tree }}(t)$.

Before proving this theorem let us recall the following definition valid in the real and complex cases.

Definition 2.7 (backward Lyapunov stable) $f$ is said to be backward Lyapunov stable if for every $\varepsilon>0$ there exists $\delta>0$ such that for every $z \in K, n \geq 0$ and $W=$ Comp $_{z} f^{-n}\left(B\left(f^{n}(z), \delta\right)\right.$ ) (the balls and components in $\mathbb{R}$ or $\left.\mathbb{C}\right)$, diam $W<\varepsilon$.

In the sequel we call $W$ a pull-back of the interval $W_{0}=B\left(f^{n}(z), \delta\right)$ for $f^{n}$, containing $z$. We use the term a pull-back for $f^{n}$ also for every component $W$ intersecting $K$, non-degenerate (i.e. not one-point), for $W_{0}$ being any interval (open, closed or open-closed) intersecting $K$. Notice that $f^{n}$ need not map $W$ onto $W_{0}$ in the case its interior contains a turning critical point for $f^{n}$.

In the real case this property always holds in absence of neutral periodic points, see [16, Lemma 2.10].

Only $\varepsilon<\varepsilon_{0}=\operatorname{dist}(K, \partial \mathbf{U})$ are considered (so the pull-backs are not "truncated" by $\mathbf{U})$.

Remark 2.8 In fact in the real case the assumption that the topological entropy of $\left.f\right|_{K}$ is positive is not needed for backward Lyapunov stability. If the entropy is positive then a stronger so-called backward asymptotic stability holds, namely the lengths of all components of $f^{-n}\left(W_{0}\right)$ intersecting $K$ converge uniformly to 0 as $n \rightarrow \infty$. See [16, Lemma 2.10 and Remark 2.11].

Definition 2.9 Let $W_{0}$ be an arbitrary interval intersecting $K$ see Definition 2.7. Define $^{3}$

$$
P\left(W_{0}, t\right)=\limsup _{n \rightarrow \infty} \frac{1}{n} \log \sum_{W_{n}}\left(\operatorname{diam} W_{n}\right)^{t}
$$

with summing over all $W_{n}$ being pull-backs of $W_{0}$ for $f^{n}$.

A part of our proof of Theorem 2.6 will be contained in the following

Lemma 2.10 For every $\alpha>0$ and $t>0$ there exists $\delta>0$ and $C>0$ such that for every $W_{0}$ shorter than $\delta, z \in K \cap \mathrm{cl} W_{0}$, every positive integer $n$ and every pull-back $W_{n}$ and $y \in \mathrm{cl} W_{n} \cap f^{-n}(z)$ one has

$$
\left|\left(f^{n}\right)^{\prime}(y)\right|^{-t} \geq C(\exp -\alpha n)\left(\frac{\operatorname{diam} W_{n}}{\operatorname{diam} W_{0}}\right)^{t} .
$$

\footnotetext{
3 This notion has been singled out in the revised version of this paper on a suggestion of a referee.
} 
If the end points of $W_{0}$, denoted by $z_{0}$ and $z_{0}^{\prime}$, belong to $K$ then

$$
P\left(W_{0}, t\right) \leq \max \left\{P_{\text {tree }}\left(z_{0}, t\right), P_{\text {tree }}\left(z_{0}^{\prime}, t\right)\right\}+\alpha .
$$

Proof Fix $\delta_{0}>0$ small enough that for every interval $W_{0}$ intersecting $K$ such that diam $W_{0} \leq \delta_{0}$ all pull-backs $W_{n}$ for iterates of $f$ are so short, that all pull-backs of $2 W_{n}$ (the interval twice longer than $W_{n}$, with the same origin) for all iterates of $f$ are shorter than $\varepsilon_{0}$. Take an arbitrary $\varepsilon<\varepsilon_{0}$ and consider $\delta \leq \delta_{0}$, both $\delta$ and $\varepsilon$ to be specified later on.

We use a procedure by Rivera-Letelier [20], see also [4, Appendix C] ${ }^{4}$ Consider an arbitrary pull-back $W_{n}$ of $W_{0}$ for $f^{n}$, where diam $W_{0} \leq \delta$. Denote by $W_{i}$ the pull-back of $W_{0}$ for $f^{i}$ containing $f^{n-i}\left(W_{n}\right)$, for each $i=0, \ldots, n$.

We consider pull-backs $\widehat{W}_{i}$ of $\widehat{W}_{0}=2 W_{0}$ for $f^{i}$ containing respective $W_{i}$ for $i=1,2, \ldots, i_{1}$ where $i=i_{1}$ is the least integer not exceeding $n$ such that $\widehat{W}_{i}$ captures a critical point, or just $n$ if such $i$ does not happen.

Next if $i_{1}=n$ we end our procedure. Otherwise, if $i_{1}<n$, we consider pullbacks $\widehat{W}_{i}^{1}$ of $\widehat{W}^{1}:=2 W_{i_{1}}$ containing respective $W_{i_{1}+i}$ for $f^{i}$ for $i=1,2, \ldots$ until for $i=i_{2}-i_{1}, \widehat{W}_{i}^{1}$ captures a critical point for the first time. Next we pull back $\widehat{W}^{2}:=2 W_{i_{2}}$ etc. until certain $i_{k}=n$.

Now due to bounded distortion between consecutive captures of critical points, more precisely for $f^{i_{s}-i_{s-1}-1}$ on $f\left(W_{i_{s}}\right)$ for $s=1, \ldots, k-1$ and similarly for $s=k$ in case $\widehat{W}_{i_{k}-i_{k-1}}^{k-1}$ contains a critical point or for $f^{i_{k}-i_{k-1}}$ on $W_{i_{k}}$ otherwise, we get for all $x \in W_{i_{s}-1}$ :

$$
\left|\left(f^{i_{s}-i_{s-1}-1}\right)^{\prime}(x)\right| \leq \text { Const } \frac{\operatorname{diam} W_{i_{s-1}}}{\operatorname{diam} W_{i_{s}-1}}
$$

and a similar bound for $x \in W_{i_{k}}$ and $\left|\left(f^{i_{k}-i_{k-1}}\right)^{\prime}(x)\right|$ in the latter non-critical case.

Also the following standard inequality holds for every $x \in W_{i}$ :

$$
\left|f^{\prime}(x)\right| \leq \text { Const } \frac{\operatorname{diam} W_{i-1}}{\operatorname{diam} W_{i}},
$$

see e.g. [4, Lemma 3.14].

Clearly for each critical point for $\delta$ small enough $\varepsilon$ is small enough that the differences of times of consecutive captures of it are bounded below by a constant arbitrarily large (this is true due to absence of attracting periodic orbits, see [12, Section 3]).

Combining these inequalities together and using the latter observation we finally get (2.5) for every $y \in W_{n}$.

Now for each $W_{n}$ denote its end points by $z_{n}$ and $z_{n}^{\prime}$. They belong to $f^{-n}\left(\left\{z_{0}, z_{0}^{\prime}\right\}\right)$.

Summing up the right hand sides of (2.5) over all $W_{n}$, taken into account that each $z_{n}$ (and $z_{n}^{\prime}$ ) can appear at most twice (as a boundary point of two adjacent $W_{n}$ 's, we conclude (2.6), provided that for each $W_{n}$ at least one of its two ends, $z_{n}$ or $z_{n}^{\prime}$, belongs to $K$. The latter is really the case:

\footnotetext{
${ }^{4}$ Compare with the related procedure in [4, Subsection 3.7 and Proposition 3.19].
} 
Lemma 2.11 For $(f, K)$ as in Theorem 2.6, for every interval $W_{0}$ short enough with end points in $K$, not in the forward trajectory of any turning critical point and for every component $W_{n}$ of $f^{-n}\left(W_{0}\right)$ intersecting $K$ (a pull-back), at least one of its end points belongs to $K$.

Proof Compare the proof of $\left[4\right.$, Lemma 3.2]. Write $W_{0}=\left[z_{0}, z_{0}^{\prime}\right]$ (we can assume it is closed) and $W_{n}=\left[z_{n}, z_{n}^{\prime}\right]$.

Take an arbitrary repelling periodic not post-critical point $p \in K$.

To simplify notation we can assume that $p$ is a fixed point for $f$. Choose a backward trajectory $\left(y_{0}, y_{1}, \ldots\right)$ of $p$ so that both $z_{0}$ and $z_{0}^{\prime}$ are its limit points.

Let $r_{p}$ be such that there exists a branch $g$ of $f^{-1}$ with $g(p)=p$, mapping $B\left(p, r_{p}\right)$ into itself, with its iterates uniformly converging to $p$.

Consider an arbitrary point $w \in W_{n} \cap K$. Choose $w_{N_{p}} \in f^{-N_{p}}(w) \cap B\left(p, \frac{1}{2} r_{p}\right)$. By backward Lyapunov stability if $W_{0}$ is short enough all its pull-backs are shorter than $\frac{1}{2} r_{p}$. Hence $W_{n+N_{p}}$, the pull-back of $W_{n}$ containing $w_{N_{p}}$, is contained in $B\left(p, r_{p}\right)$.

Choose the intervals $B=B\left(z_{0}, \xi\right)$ and $B^{\prime}=B\left(z_{0}^{\prime}, \xi\right)$ so short that the pull-back $B_{n+N_{p}}$ of a one of them, say of $B$, containing $z_{n+N_{p}}$ being a boundary point of $W_{n+N_{p}}$, is contained in $B\left(p, r_{p}\right)$ and $f^{n+N_{p}}$ has no turning critical points in it. Next choose $r^{\prime}$ and $n^{\prime}$ such that a pull-back $W^{\prime \prime}$ of $B\left(p, r^{\prime}\right)$ for $f^{n^{\prime}}$ is in $B$. Finally choose $m$ such that $g^{m}\left(B\left(p, r_{p}\right)\right) \subset B\left(p, r^{\prime}\right)$. So the adequate branch $G$ of $f^{-\left(n+N_{p}+m+n^{\prime}\right)}$ maps $B$ into itself, so a corresponding fixed point $p_{\xi}$ for $f^{n+N_{p}+m+n^{\prime}}$ exists in $B$.

Suppose that $f^{N_{p}}\left(z_{n+N_{p}}\right)=z_{n}$. We have $f^{n}\left(z_{n}\right)=z_{0}$. A part of the periodic trajectory of $p_{\xi}$ shadows the backward trajectory $\left(z_{0}, \ldots, z_{n+N_{r}}\right)$. By the weak isolation property $p_{\xi} \in K$. The shadowing error tends to 0 as $\xi \rightarrow 0$. Thus $z_{n} \in K$. Alternatively we prove that $z_{n}^{\prime} \in K$.

This ends the proof of Lemma 2.11 and therefore the proof of Lemma 2.10.

Remark 2.12 In fact asymptotic backward stability allows to get rid of $\alpha$ in (2.6).

Proof of Theorem 2.6 The inequality $P_{\text {tree }}(z, t) \geq P_{\text {hyp }}\left(f, \phi_{t}\right)$ is obviously true for every $z \in K$, under a mild non-exceptionality condition, weaker than safe, see [16, Lemma 4.4].

The opposite inequality was proved in [16] for $z$ safe satisfying additional assumptions, mentioned at the beginning of this subsection. So to prove this opposite inequality for every $z$ safe, it is enough to prove the independence of $P_{\text {tree }}(z, t)$ of $z$ for all $z$ safe. For this end it is enough to prove that for all $w, z \in K$ both being safe,

$$
P_{\text {tree }}(w, t) \leq P_{\text {tree }}(z, t)
$$

Then the equality follows, since we can interchange the roles of $w$ and $z$ in this inequality.

It follows from the topological transitivity of $\left.f\right|_{K}$ and the compactness of $K$ that given any $\delta^{\prime}>0$ there exists $N\left(\delta^{\prime}\right)$ such that $A=A\left(z, \delta^{\prime}\right):=\bigcup_{j=0, \ldots, N} f^{-j}(z) \cap K$ is $\delta^{\prime}$-dense in $K$ (i.e. $\bigcup_{y \in A} B\left(y, \delta^{\prime}\right) \supset K$; in other words $\left(0, \delta^{\prime}\right)$-spanning), see [16, Remark 2.6, Proposition 2.4]. 
Take $\varepsilon>0$ which satisfies the weak isolation condition 2.5. Take an arbitrary $\alpha>0$ and choose $\delta<\delta_{0}$ as in Lemma 2.10, with $\delta_{0}$ as in the beginning of Proof of Lemma 2.10 .

We have two cases:

Case 1. There exist $z_{0}, z_{0}^{\prime} \in A\left(z, \delta^{\prime}\right)$ such that $z_{0} \leq w \leq z_{0}^{\prime}$ and $\left|z_{0}-z_{0}^{\prime}\right| \leq \delta$. Write $W_{0}=\left[z_{0}, z_{0}^{\prime}\right]$. We can assume that none of $z_{0}$ or $z_{0}^{\prime}$, is postcritical since otherwise the right hand side of (2.6) is infinite, so (2.6) is obviously true. Then, by Lemma 2.11, for an arbitrary $n$ and pull-back $W_{n}$, there exists $z_{n} \in f^{-n}\left(\left\{z_{0}, z_{0}^{\prime}\right\}\right) \cap \partial W_{n} \cap K$. Hence, by Lemma 2.10 for $y=z_{n}$

$$
\left|\left(f^{n}\right)^{\prime}\left(z_{n}\right)\right|^{-t} \geq C(\exp -\alpha n)\left(\frac{\operatorname{diam} W_{n}}{\operatorname{diam} W_{0}}\right)^{t} .
$$

Take an arbitrary $\xi>0$. Since $w$ is safe, see Definition 2.1, there exists $c(w, \xi)>0$ such that for every $w_{n} \in W_{n} \cap f^{-n}(w) \cap K$ for the set $V_{0}:=B(w, c(w, \xi) \exp (-\xi n)) \cap$ $W_{0}$ and for the pull-back $V_{n}$ of $V_{0}$ for $f^{n}$ containing $w_{n}$, the map $f^{n}$ is invertible of bounded distortion on $V_{n}$. Hence, for all $n$ large enough,

$$
\left(\operatorname{diam} V_{n}\right)^{t} \geq \exp (-2 t \xi n)\left|\left(f^{n}\right)^{\prime}\left(w_{n}\right)\right|^{-t}
$$

Hence

$$
\left|\left(f^{n}\right)^{\prime}\left(z_{n}\right)\right|^{-t} \geq(\exp -2(\alpha+t \xi) n)\left|\left(f^{n}\right)^{\prime}\left(w_{n}\right)\right|^{-t} .
$$

Summing over all $n$ 'th preimages $w_{n}$ of $w$ in $K$, taking in account that the number of $w_{n}$ 's can be at most exp $\alpha_{1} n$ in each pull-back $W_{n}$, for $n$ large enough, for an arbitrary $\alpha_{1}>0$ for appropriate $\delta$ (similarly to $\alpha$ in Proof of Lemma 2.10), finally passing with $\alpha, \xi$ and $\alpha_{1}$ to 0 , gives the demanded estimate (2.8).

More precisely:

$$
\begin{aligned}
Q_{n}(w, t) & \leq 2 Q_{n}\left(z_{0}, t\right)+2 Q_{n}\left(z_{0}^{\prime}, t\right) \\
& \leq 4 \max _{j=0, \ldots, N} Q_{n+j}(z, t) L^{j t} \leq 4 Q_{n+N}(z, t) L^{N t}
\end{aligned}
$$

for $L=\sup \left|f^{\prime}\right|>1$. Acting with $\frac{1}{n} \log$ and passing with $n$ to $\infty$ in limsup we get the inequality (2.8) for $w$ and $z$ hence after the interchanging their roles, the equality, as mentioned already at the beginning of the proof.

Similarly we obtain the equality of lower limits, writing

$$
Q_{n}(z, t) \geq \frac{1}{4} L^{-N t} Q_{n-N}(w, t) .
$$

But, similarly to upper limits, they coincide with $P_{\text {hyp }}(t)$ for $z$ safe, safe forward and hyperbolic, see the beginning of this section and [16, Remark 4.5] and [19, Remark 12.5.18] in the complex case. Hence limsup and liminf coincide and are equal to $P_{\text {hyp }}(t)$ for every $z$ safe. 
Case 2. The safe point $w \in K$ is not between two points $z_{0}, z_{0}^{\prime}$, in the notation of Case 1 . We assume $\delta^{\prime} \leq \delta / 4$. Then the interval $\left(w-\delta+2 \delta^{\prime}, w-\delta^{\prime}\right)\left(\right.$ or $\left(w+\delta^{\prime}, w+\right.$ $\left.\delta-2 \delta^{\prime}\right)$ ) is disjoint from $K$. Call any component of $\mathbb{R} \backslash K$ of length at least $\delta / 4$ a large gap. Thus $w$ is $\delta^{\prime}$-close to a large gap.

By the boundness of $K$ there are at most a finite number $\Gamma_{\delta}$ of large gaps. Denote the union of the of the large gaps by $G$ and consider the set of the boundary points of them by $\partial G$. For $\delta^{\prime}$ satisfying additionally

$$
L \delta^{\prime}+\delta^{\prime}<\operatorname{dist}(f(\partial G) \backslash \partial G, \partial G) .
$$

implying $\bigcup_{x \in \partial G, f(x) \notin \partial G} B\left(f(x), \delta^{\prime}\right) \cap B\left(\partial G, \delta^{\prime}\right)=\emptyset$, we conclude that either for some $m: 0<m \leq 2 \Gamma_{\delta}, f^{m}(w)$ satisfies the assumption of Case 1 (is between $z_{0}, z_{0}^{\prime}$ ), or all $f^{j}(w)$ for $0 \leq j<2 \Gamma_{\delta}$ are $\delta^{\prime}$ close to $\partial G$. Notice that due to (2.10) the point in $\partial G$ close to $w$ is pre-periodic, i.e. its image under some $f^{j}$ is periodic for $f$.

Since $\delta^{\prime}$ can be taken arbitrarily small, only the case $f^{j}(w) \in \partial G$ for all $0 \leq j<$ $2 \Gamma_{\delta}$ is to be considered. Hence, it is sufficient to consider $w \in \partial G$ with forward orbit also in $\partial G$ and pre-periodic itself, and of length bounded by $2 \Gamma_{\delta}$.

Then use $\widehat{z} \in f^{-\kappa n}(z)$ which is $\exp -\eta n$ close to $w$ i.e. in a "safe" ball, for $0<\eta<\kappa \chi$, where $\chi$ is Lyapunov exponent at $w$. Taking $\kappa$ arbitrarily small (positive) we can replace $z$ by $\widehat{z}$ when comparing $Q_{n}$ 's in the tree pressures at $z$ and $w$. We use $\left|f^{\prime}\right| \leq L$ and $t \geq 0$. We use also the fact that by the safety condition the distortion $\left|\left(f^{n}\right)^{\prime}\left(\widehat{z}_{n}\right)\right| /\left|\left(f^{n}\right)^{\prime}\left(w_{n}\right)\right|$ is uniformly bounded for $w_{n}$.

(This allows not to use $\widehat{z}^{\prime} \in f^{-k}(z)$ on the other side of $w$ maybe not existing for $k$ of order at most $\kappa n)$.

\subsection{On the weak isolation condition in absence of weakly exceptional points}

Notice that proving $z_{n} \in K$ in Lemma 2.11, we used the existence of $z=z_{n+N_{r}}$ close to periodic $p \in K$ such that $f^{N_{r}}(z)=z_{n} \in \partial W_{n}$. The other end of $W_{n}$, denoted by $z_{n}^{\prime}$ may not belong to $K$.

An example is $f(x)=a x(1-x)$ for $a<4$ close to 4 , on a neighbourhood $U$ of $\widehat{I}=\left[f^{2}(1 / 2), f(1 / 2)\right]$. Then points $z_{n}^{\prime}$ slightly to the left of $f^{2}(1 / 2)$, in the boundary of respective $W_{n} \ni f^{2}(1 / 2)$, are not in $\widehat{I}$, hence not in $K$.

In fact they have no preimages in $\widehat{I}$. The pull-back of $W_{n}$ for $f$ intersecting $\widehat{I}$ does not contain any $f$-preimage of $z_{n}^{\prime}$.

Notice again that in Proof Lemma 2.11, we used only those $z_{i}$ which are boundary points of pull-backs of $\left[z_{0}, z_{0}^{\prime}\right]$ intersecting $K$, more precisely: containing $w_{i} \in K$.

In Proof of Lemma 2.11, to know that $z_{n}$ or $z_{n}^{\prime}$ belongs to $K$ we could refer to [4, Corollary 3.3] in the form of Proposition 2.14 below (interesting in itself), under the additional assumption, see [4, Subsection 1.4], that no point in $\partial \widehat{I}$ is weakly $\Sigma$ exceptional, for $\Sigma$ being the set of all turning critical points.

Definition 2.13 Given an arbitrary finite set $\Sigma \subset K$, we call a nonempty set $E \subset K$ weakly $\Sigma$-exceptional, if $E$ is non-dense in $K$ and satisfies 


$$
\left(\left.f\right|_{K}\right)^{-1}(E) \backslash \Sigma \subset E .
$$

We call $x \in K$ weakly $\Sigma$-exceptional if it is contained in a weakly $\Sigma$-exceptional set.

Proposition 2.14 (On K-homeomorphisms) Let $(f, K) \in \mathscr{A}_{+}$satisfy weak isolation condition. Let $W$ be an arbitrary interval sufficiently short (closed, half-closed or open), not containing in its closure weakly $\Sigma$-exceptional points for $\Sigma$ being the set of turning critical points in $\partial \widehat{I}$, such that $f$ is monotone on $W$ and $\mathrm{cl} W \cap K \neq \emptyset$. Then $\left.f\right|_{W}$ is a $K$-homeomorphism, that is $f(W \cap K)=f(W) \cap K$.

Proof It is sufficient to consider $W$ closed. The assertion of the Proposition follows for $W^{\prime}:=W \cap \widehat{I}=W \cap \widehat{I}_{j}$ by the maximality of $K$ (notice that $W$ short enough intersects only one interval $\widehat{I}_{j}$ ). By definition $W^{\prime \prime}:=W \backslash W^{\prime}$ is disjoint from $K$. For $W$ short enough $W^{\prime \prime}$ has one component or it is empty (we use the assumption that the family $\widehat{I}_{j}$ is finite). Suppose it is non-empty. Denote the boundary point of $\widehat{I}_{j}$ belonging to $\mathrm{cl} W^{\prime \prime}$ by $a$. The case $f\left(W^{\prime \prime}\right)$ intersects $K$, but $f(a)$ is not a limit point of $f\left(W^{\prime \prime}\right) \cap K$ can be eliminated by considering $W$ short enough.

Therefore we need only to consider the case $f(a)$ is an accumulation point of $f\left(W^{\prime \prime}\right) \cap K$ (in particular $\left.f\right|_{K}$ is not open at $a$ ). In this case however there exists a periodic orbit $Q$ passing through $W^{\prime \prime}$ arbitrarily close to $K$. The proof is the same as the proof of [4, Lemma 3.2] and similar to the proof of Theorem 2.6. Briefly: we choose a repelling periodic orbit $\mathscr{O} \subset K$. Next choose a backward trajectory $\left(y_{0}, y_{1}, \ldots\right)$ of a point $p \in \mathscr{O}$ with a limit point in $f\left(W^{\prime \prime}\right) \cap K$ and a backward trajectory $\left(z_{0}, z_{1}, \ldots\right)$ of $a$ converging to $Q$. This allows us to find a backward trajectory of $W^{\prime \prime}$ at a time $n$ approaching to $\mathscr{O}$ along $z_{j}$ and next at a time $m$ being in $f\left(W^{\prime \prime}\right)$. So $W^{\prime \prime}$ after the time $n+m+1$ enters itself. Hence there exists a branch of $f^{-(m+n+1)}$ mapping $W^{\prime \prime}$ into itself, yielding the existence of $Q$.

So $Q$ is in $K$ by the weak isolation condition. We obtain a point in $K \cap W^{\prime \prime}$, a contradiction.

Remark that in the example $f(x)=a x(1-x)$ discussed above the assumption of the lack of weakly $\Sigma$-exceptional points does not hold and the assertion of Proposition 2.14 fails for $W=\left[f^{2}(1 / 2)-\delta, f^{2}(1 / 2)\right]$.

\section{Geometric pressure via spanning sets: the complex case}

In the real case in the previous section we used the property: backward Lyapunov stability, Definition 2.7. In the complex case this property need not hold.

So the following weaker version occurs useful.

Definition 3.1 A rational mapping $f: \widehat{\mathbb{C}} \rightarrow \widehat{\mathbb{C}}$ is said to be weakly backward Lyapunov stable wbls, if for every $\delta>0$ and $\varepsilon>0$ for all $n$ large enough and every disc $B=B(x, \exp -\delta n)$ centered at $x \in J(f)$, for every $0 \leq j \leq n$ and every component $V$ of $f^{-j}(B)$ it holds that diam $V \leq \varepsilon$.

Denote $P_{\text {spanning }}\left(\left.f\right|_{K}, \phi_{t}\right)$ by $P_{\text {spanning }}(t)$, both in the real and complex case. The following is the main theorem in this section. 
Theorem 3.2 For every rational mapping $f: \widehat{\mathbb{C}} \rightarrow \widehat{\mathbb{C}}$ of degree at least 2 and for every $t>0, P_{\text {spanning }}(t) \geq P_{\text {tree }}(t)$ holds. If $f$ is wbls then the opposite inequality also holds, hence

$$
P_{\text {spanning }}(t)=P_{\text {tree }}(t)
$$

Proof I. First we prove $P_{\text {spanning }}(t) \leq P_{\text {tree }}(t)$. This is the CONSTRUCTION part of the proof, where we construct an $(n, \varepsilon)$-spanning set not carrying much more "mass" than $f^{-n}\left(\left\{z_{0}\right\}\right)$. This corresponds to the right hand side inequality in (1.4), where we can just consider maximal $(n, \varepsilon)$-separated sets as the $(n, \varepsilon)$-spanning sets to be constructed.

Fixed an arbitrary $\varepsilon>0$ and $\delta>0$, by the property wbls we have for $n$ large enough for every $x \in J(f)$ and every pull-back $V$ of $B(x, \exp (-n \delta / 2))$ for $f^{j}, j=0, \ldots, n$, $\operatorname{diam} V<\varepsilon$.

Denote $\mathscr{B}:=\bigcup_{c \in \operatorname{Crit}(f) \cap J(f)} \bigcup_{j=1, \ldots, n} B(c, j)$, where $B(c, j):=B\left(f^{j}(c), r\right)$ where $r:=\exp (-n \delta)$ and $\delta=\xi$ as in the safety assumption.

We can easily find a set $X \subset J(f) \backslash \mathscr{B}$ which is $(0, r / 2)$-spanning for $\rho$ the standard metric on the Riemann sphere, i.e. the set $B(X, r / 2)$ covers $J(f) \backslash \mathscr{B}$, and $\# X \leq$ Const $\exp 2 n \delta$.

Let $B^{1}, B^{2}, \ldots B^{N}$ be all the components of $\mathscr{B}$.

Assume first for simplicity that $J(f)$ is connected.

Clearly for every $1 \leq k \leq N$, diam $B^{k} \leq 2 r \cdot n \#(\operatorname{Crit}(f) \cap J(f))$. By the connectedness of $J(f)$, there exists $x^{k} \in \partial B^{k} \cap J(f)$ if $n$ is large enough. For $n$ large we have also diam $B^{k}<\exp (-n \delta / 2)$. Hence the diameters of all pull-backs $B_{j}^{k}$ of $B^{k}$ for $f^{j}, j=1, \ldots, n$ are less than $\varepsilon$. Let $\widehat{X}=X \cup \bigcup_{k}\left\{x^{k}\right\}$. Then $Y=f^{-n}(\widehat{X})$ is $(n, \varepsilon)$-spanning. This is so because the diameters of all the pull-backs $V$ and $B_{n}^{k}$ in the metric $\rho_{n}$ are less than $\varepsilon$.

We have for an arbitrary $\xi>0$

$$
\sum_{y \in Y}\left|\left(f^{n}\right)^{\prime}(y)\right|^{-t}=\sum_{z \in \widehat{X}} Q_{n}(z, t) \leq \#(\widehat{X}) \exp n\left(P_{\text {tree }}(t)+\xi\right)
$$

for all $n$ large enough. This uses the fact that the convergence in (2.1) is uniform for all $z$ safe with the same $\delta$, see Lemma 3.5 below. (Here we consider $z \in \widehat{X}$ depending on $n$, so we abuse the terminology; we consider safe for each $n$ separately, just satisfying (2.2).) Considering $n \rightarrow \infty$ and passing with $\delta$ and $\varepsilon$ to 0 , we end the proof in the connectedness case.

Now consider the general case, allowing $J(f)$ being disconnected.

Definition 3.3 A compact set $X \subset \mathbb{C}$ in the complex plane is said to be uniformly perfect if there exists $M>0$ such that there is no annulus $D \subset \mathbb{C}$ of modulus bigger than $M$, separating $X$. Equivalently, there exists $M>0$ for which there is no $A=\left\{z \in \mathbb{C}: r_{1}<\left|z-z_{0}\right|<r_{2}\right\}$ such that $\log \frac{r_{2}}{r_{1}}>M$, and $X \cap\left\{\left|z-z_{0}\right| \leq r_{1}\right\} \neq \varnothing$ and $X \cap\left\{\left|z-z_{0}\right| \geq r_{2}\right\} \neq \emptyset$ and $A \cap X=\emptyset$. 
Lemma 3.4 Let $X \subset \mathbb{C}$ be a compact uniformly perfect set. Then there exists $\kappa>0$ such that for every $0<a \leq 1$, every $m$ large enough and every $\widehat{x} \in X$ there exists in the Euclidean metric an $\exp (-m)$-separated set $X_{m, a} \subset B(\widehat{x}, \exp -(1-a) m) \cap X$ such that \# $X_{m, a} \geq \exp \kappa a m$.

Proof ${ }^{5}$ We can assume diam $X \geq 2$. If $X$ is uniformly perfect with constant $M$, then for every $x \in X$ and $i \geq 0$ such that $(3 i+1) M<a m$ we can find $x_{i}^{\prime} \in X$ such that

$$
\exp (-m+3 i M) \leq\left|x-x_{i}^{\prime}\right| \leq \exp (-m+(3 i+1) M)
$$

Now we define $X_{m, a}$. Each of its elements will be encoded by a block of 0's and 1's of length $\mathcal{I}+1$ where $\mathcal{I}:=[\mathrm{am} / 3 \mathrm{M}]-1$ where $[\cdot]$ means the integer part, and denoted $x\left(v_{0}, \ldots, v_{\mathcal{I}}\right)$, where $v_{j}=0$ or 1 . We define these points by induction using codings of length $1,2, \ldots$ and finally $\mathcal{I}+1$ which will be our final choice.

For $x=\widehat{x}$ set $x_{0}(0)=x$ and $x_{0}(1)=(x)_{\mathcal{I}}^{\prime}$. The subscript at $x$ denotes the length of the block of the coding symbols minus 1 , here length 1 . Having defined $x_{i}\left(v_{0}, \ldots, v_{i}\right)$ define

$$
\begin{aligned}
& x_{i+1}\left(v_{0}, \ldots, v_{i}, 0\right)=x_{i}\left(v_{0}, \ldots, v_{i}\right) \text { and } \\
& x_{i+1}\left(v_{0}, \ldots, v_{i}, 1\right)=\left(x_{i}\left(v_{0}, \ldots, v_{i}\right)\right)_{\mathcal{I}-(i+1)}^{\prime}
\end{aligned}
$$

Notice that all the points $x\left(v_{1}, \ldots, v_{\mathcal{I}}\right)$ are within the distance at least $\exp (-m)$ from each other. Indeed, if $0 \leq i \leq \mathcal{I}$ is the first index with digits $v_{i}$ different for two such points $y=x\left(v_{0}, \ldots, v_{\mathcal{I}}\right)$ and $z=x\left(v_{0}^{*}, \ldots, v_{\mathcal{I}}^{*}\right)$, then, say $v_{i}=0, v_{i}^{*}=1$, by construction, setting $x_{-1}=x_{0}$ for $i=0$,

$$
\begin{aligned}
& |y-z| \geq \mid x_{i}\left(v_{0}, \ldots, v_{i-1}, 0\right) \\
& \quad-\left(x_{i}\left(v_{0}, \ldots, v_{i-1}, 1\right)|-| y-x_{i}\left(v_{0}, \ldots, v_{i-1}, 0\right)|-| z-x_{i}\left(v_{0}, \ldots, v_{i-1}, 1\right) \mid\right. \\
& \geq \exp (-m+3(\mathcal{I}-i) M)-2 \sum_{s=i+1}^{\mathcal{I}} \exp (-m+3(\mathcal{I}-s) M) \\
& \geq \exp (-m)\left(\exp 3(\mathcal{I}-i) M-2 \frac{\exp (3(\mathcal{I}-i) M)-1}{\exp (3 M)-1}\right) \geq \exp (-m)
\end{aligned}
$$

for $M \geq 1$.

Thus, define $X_{m, a}=\left\{x\left(v_{0}, \ldots, v_{\mathcal{I}}\right)\right\}$. Notice that

$$
\# X_{m, a}=2^{[a m / 3 M]+1}=\exp ((\log 2)[a m / 3 M]+1) \geq \exp \kappa a m
$$

with $\kappa$ arbitrarily close to $(\log 2) / 3 M$ for am large enough. The corresponding assertion of the lemma is proved.

\footnotetext{
${ }^{5}$ We provide the proof for completeness. Its arguments were known before, and used to prove that the uniform perfectness implied the positivity of Hausdorff $h$-content for an $h>0$. See [7] and [23, Proof of Theorem 3.1].
} 
Finally notice that for each $y \in X_{m, a}$

$$
|\widehat{x}-y|<\exp (-m+(3 \mathcal{I}+2) M) \leq \exp (-m+a m)=\exp -(1-a) m
$$

Continuation of Proof of Theorem 3.2 We deal now with the non-connected $J(f)$ case. Let $m:=-\log r$, i.e. $\exp -m=r$ and $m=n \delta$. Then by Lemma 3.4 applied to $a=1 / 2$ for each $x \in J(f)$ there is a set $X(x)$ of at least $\exp \kappa m / 2=\exp \kappa n \delta / 2$ of $r$-separated points in $J(f) \cap B(x, \exp -m / 2)$ in particular in $B(x, \exp -n \delta / 2)$. Since $n \#(\operatorname{Crit}(f) \cap J(f)) \ll \exp \kappa n \delta$ for $n$ large enough, then for each $x=f^{j}(c), c \in$ $\operatorname{Crit}(f) \cap J(f), j=1, \ldots, n$, there is a point $\widehat{x}$ in $B(x, \exp -n \delta / 2) \backslash \mathscr{B}$. Now we repeat the proof as in the connected $J(f)$ case, with $\widehat{x}$ playing the role of $x^{k}$.

II. Now we prove the opposite inequality, namely

$$
P_{\text {spanning }}(t) \geq P_{\text {tree }}(t) \text {. }
$$

In fact we shall prove

$$
P_{\text {spanning }}(t) \geq P_{\text {hyp }}(t)
$$

which is enough due to Theorem (2.2).

By [18, Proposition 2.1] for every $\xi>0$ and $t>0$ there exists an $f$-invariant isolated hyperbolic set $X \subset J(f)$ such that

$$
P\left(\left.f\right|_{X},\left.\phi_{t}\right|_{X}\right) \geq P_{\text {hyp }}(t)-\xi
$$

Then for every $\varepsilon>0$ small enough for every $n \geq 0$ large enough there exists an $(n, 2 \varepsilon)$-separated set $X_{n} \subset X$ such that

$$
\sum_{y \in X_{n}}\left|\left(f^{n}\right)^{\prime}(y)\right|^{-t} \geq \exp \left(P_{\operatorname{sep}}\left(\left.f\right|_{X},\left.\phi_{t}\right|_{X}\right)-\xi\right)
$$

Therefore for every $(n, \varepsilon)$-spanning set $Y_{n} \subset J(f)$ for every $y \in X_{n}$, there exists $y^{\prime} \in Y_{n}$ which is $(n, \varepsilon)$-close to $y$.

Hence by triangle inequality the selection $y \mapsto y^{\prime}$ is injective. By the hyperbolicity of $X$, if $\varepsilon$ is small enough, there is a constant $C$ such that for every $n$ and $y \in X_{n}$ it holds that $\left|\left(f^{n}\right)^{\prime}\left(y^{\prime}\right)\right| /\left|\left(f^{n}\right)^{\prime}(y)\right| \leq C$. This, after passing to limits, accounting (3.4), proves $P_{\text {spanning }}(t) \geq P_{\text {sep }}\left(\left.f\right|_{X},\left.\phi_{t}\right|_{X}\right)-\xi$. Hence letting $\xi \rightarrow 0$ and choosing appropriate $X$, using (3.3), we obtain $P_{\text {spanning }}(t) \geq P_{\text {hyp }}(t)$.

We considered here all $(n, \varepsilon)$-spanning sets, so it is natural to call this Part II of the proof the ALL part. Notice that this corresponds to the left hand side inequality in (1.4).

To end this section let us provide the lemma we have already referred to 
Lemma 3.5 For every $t>0$ and $\gamma>0$ there exists $\delta_{0}>0$ and $n_{0} \geq 0$ such that for every $0<\delta \leq \delta_{0}$ and $n \geq n_{0}$ and every $z_{1}, z_{2} \in J(f)$ such that $z=z_{i}, i=1,2$ satisfies (2.2) with $\delta=\xi$ there,

$$
\exp -n \gamma \leq \frac{Q_{n}\left(z_{1}, t\right)}{Q_{n}\left(z_{2}, t\right)} \leq \exp n \gamma
$$

holds for $Q_{n}$ defined in (2.1)

To prove this lemma we use the fact which is part of [14, Lemma 3.1] (see also [6] and [17, Geometric Lemma])

Lemma 3.6 There exists $C>0$ such that for every set $W$ of $m>0$ points in $\widehat{\mathbb{C}}$ and $0<r<1 / 2$ such that $m \geq \log 1 / r$, for every $z_{1}, z_{2} \in \widehat{\mathbb{C}} \backslash B(W, r)$ there exists a sequence of discs in the Riemann sphere metric $B_{1}=B\left(q_{1}, r_{1}\right), \ldots, B_{k}=B\left(q_{k}, r_{k}\right)$ such that for every $j=1, \ldots, k$ each $2 B_{j}:=B\left(q_{j}, 2 r_{j}\right)$ is disjoint from $W, z_{1} \in$ $B_{1}, z_{k} \in B_{k}, B_{j} \cap B_{j+1} \neq \emptyset$ for all $j=1, \ldots, k-1$ and

$$
k \leq C \sqrt{m} \sqrt{\log 1 / r} .
$$

In other words the quasi-hyperbolic distance between $z_{1}$ and $z_{2}$ in $\widehat{\mathbb{C}} \backslash W$ is bounded by Const $\sqrt{m} \sqrt{\log 1 / r}$.

Proof of Lemma 3.5 Given $n$ set $W=\bigcup_{j=1, \ldots, n} f^{j}(\operatorname{Crit}(f))$ and $m=n \# \operatorname{Crit}(f)$. Using Lemma 3.6 and Koebe distortion lemma we obtain for a sequence $s_{j} \in B_{j} \cap B_{j+1}$ for $j=1, \ldots, k-1$ and $s_{0}=z_{1}, s_{k+1}=z_{2}$, and for a distortion constant $C_{\text {Dist }}>0$

$$
\frac{Q_{n}\left(s_{j}, t\right)}{Q_{n}\left(s_{j+1}, t\right)} \leq C_{\text {Dist }}^{t},
$$

hence

$$
\frac{Q_{n}\left(z_{1}, t\right)}{Q_{n}\left(z_{2}, t\right)} \leq C_{\text {Dist }}^{t(k+1)} .
$$

Hence, for $r=\exp -n \delta$, due to

$$
k \leq C \sqrt{n \# \operatorname{Crit}(f)} \sqrt{n \delta}=C^{\prime} n \sqrt{\delta}
$$

for $C^{\prime}=C \sqrt{\# \operatorname{Crit}(f)}$, we obtain

$$
\frac{Q_{n}\left(z_{1}, t\right)}{Q_{n}\left(z_{2}, t\right)} \leq C_{\mathrm{Dist}}^{t\left(C^{\prime} n \sqrt{\delta}+1\right)} \leq \exp n \gamma
$$

for $\delta$ small enough and $n$ satisfying (2.2) and large enough to satisfy the latter inequality. 


\section{Weak backward Lyapunov stability and further corollaries in the complex case}

For every $x \in \widehat{\mathbb{C}}$ and a rational mapping $f: \widehat{\mathbb{C}} \rightarrow \widehat{\mathbb{C}}$ define the lower Lyapunov exponent by

$$
\underline{\chi}(x):=\liminf _{n \rightarrow \infty} \frac{1}{n} \log \left|\left(f^{n}\right)^{\prime}(x)\right| .
$$

Let us start with the following

Proposition 4.1 For every rational mapping $f: \widehat{\mathbb{C}} \rightarrow \widehat{\mathbb{C}}$ of degree at least 2 , if for every critical point $c \in J(f)$ the lower Lyapunov exponent $\underline{\chi}(f(c))$ is non-negative, then weak backward Lyapunov stability wbls holds.

Proof Take arbitrary $\varepsilon, \delta>0$ and $x \in J(f)$, and an arbitrary $n$ large enough. Consider $B:=B(x, \exp -n \delta)$ and an arbitrary $y \in f^{-n}(x)$. For every $0<j \leq n$ consider $U_{j}=B\left(x, a_{j} \exp -n \delta\right)$, where $a_{j}=\prod_{s=1}^{j}\left(1-\frac{1}{2} s^{-2}\right)$. Let $V_{j}$ be the pull-back of $U_{j}$ for $f^{j}$ containing $f^{n-j}(y)$. Let $j=j_{1}$ be the least non-negative integer for which $V_{j+1}$ contains a critical point $c$.

Then $c \in J(f)$ if $n$ is large enough. Indeed, the only other possibility would be a critical point $c \notin J(f)$ attracted to a parabolic periodic orbit. Then however the convergence of $f^{n}(c)$ to this orbit, and moreover to $J(f)$ would be subexponential, so $f^{s}(c) \notin B(x, \exp -n \delta)$ for $s=1,2, \ldots, n$ if $n$ is large enough.

(In fact we can omit this part of the proof, since in the further considerations it will not matter whether $c$ is in $J(f)$, or is not. We shall use only $\chi(f(c)) \geq 0$, automatically true if $f^{n}(c)$ converges to a parabolic periodic orbit.)

Then, for diameters and derivatives in the spherical metric, for $j=j_{1}$ :

$$
\frac{\operatorname{diam} f\left(V_{j+1}\right)}{\operatorname{diam} U_{j+1}} \leq C_{1}(j+1)^{8}\left|\left(f^{j}\right)^{\prime}(f(c))\right|^{-1} \leq C_{2} \exp j \xi,
$$

The term $C_{1}(j+1)^{8}=C_{1}\left((j+1)^{2}\right)^{4}$ results from Koebe's distortion bounds, see e.g. [19, Lemma 6.2.3] for the spherical setting. The 'isolating annulus' is

$$
a_{j+1} \exp -n \delta<|z-x|<a_{j} \exp -n \delta .
$$

The number $\xi$ is arbitrary positive and $C_{2}$ is an appropriate constant, depending on $c$ and $\xi$, but not on $n$. The most right inequality in (4.1) follows from the assumption that $\underline{\chi}(f(c)):=\liminf _{n \rightarrow \infty} \frac{1}{n} \log \left|\left(f^{n}\right)^{\prime}(f(c))\right| \geq 0$. In the sequel we shall assume that $\bar{\xi}<\delta / 4$.

This method of controlling distortion was introduced in [13, Definition 2.3] and developed and called in [5] shrinking neighbourhoods. $j=j_{1}$ is called the first essential critical time.

Consider now $B_{0}=B(x, \kappa \exp -n \delta)$, for $0<\kappa \ll 1$ small enough that $B_{0}$ is deeply in $B\left(x, \prod_{s=1}^{\infty}\left(1-s^{-2}\right) \exp -n \delta\right)$ so that for the pull-backs $W_{t}$ of $B_{0}$ in $V_{t}$, 
for $t=1,2, \ldots, j_{1}$ we have diam $W_{t} \leq \varepsilon$. This is possible due to bounded distortion before the capture of $c$, more precisely bounded distortion of the appropriate branch $g$ of $f^{-j_{1}}$ on $\frac{1}{2 \kappa} B_{0}$ leading to $V_{j_{1}}$, for $\kappa \ll \varepsilon$.

(Alternatively one can refer to the fact that a topological annulus of a big modulus contains a geometric annulus of a big modulus).

Now notice that just for $j=j_{1} \geq N$ for a constant $N=N(\delta, \xi)$ we obtain using (4.1)

$$
\operatorname{diam} W_{j_{1}} \leq \exp \left(-n \delta+j_{1} 2 \xi\right) \leq \kappa \exp \left(-n \delta+j_{1} \delta / 2\right),
$$

which is an estimate better than by $\varepsilon$.

Hence, denoting by $\tau=\tau(c)$ the multiplicity of $f$ at any critical point $c$ and denoting the captured $c$ by $c_{1}$ we get

$$
\operatorname{diam} W_{j_{1}+1} \leq \kappa \exp \frac{1}{\tau\left(c_{1}\right)}\left(-n \delta+j_{1} \delta\right)
$$

If $j_{1}<N$ then we obtain (4.3) automatically if we replace $B_{0}$ by a disc centered at $x$ of diameter $a \kappa \exp (-n \delta)$ with $a$ small enough.

Denote $n_{1}=n-j_{1}-1$. Apply the shrinking neighbourhood procedure starting from $\widehat{B}_{1}:=B\left(f^{n_{1}}(y), \exp -n_{1} \delta / \tau\left(c_{1}\right)\right)$. Let $0<j_{2} \leq n_{1}$ be the first essential critical time, if it exists. Denote the captured critical point by $c_{2}$ (it can be different from the former $c_{1}$ ).

Denote $B_{1}=B\left(f^{n_{1}}(y), \kappa \exp -n_{1} \delta / \tau\left(c_{1}\right)\right)$. Notice that $\widehat{B}_{1} \ni B_{1} \supset W_{j_{1}+1}$. Denote the consecutive pull-backs of $B_{1}$ by $W_{1, j}$. Repeating (4.1) we obtain, analogously to (4.2), using an analog of (2.7),

$$
\begin{aligned}
& \operatorname{diam} W_{1, j_{2}} \leq C_{1}\left(j_{2}+1\right)^{8}\left|\left(f^{j_{2}}\right)^{\prime}\left(f\left(c_{2}\right)\right)\right|^{-1} \operatorname{diam} B_{1} \\
& \quad \leq \text { Const } C_{1}\left(j_{2}+1\right)^{8}\left|\left(f^{j_{2}}\right)^{\prime}\left(f\left(c_{2}\right)\right)\right|^{-1}\left|f^{\prime}\left(f^{j_{2}}\left(f\left(c_{2}\right)\right)\right)\right|^{-1} \operatorname{diam} f\left(B_{1}\right) \\
& \quad \leq C_{2} \exp \left(\left(j_{2}+1\right) \xi\right) \operatorname{diam} f\left(B_{1}\right) \\
& \quad \leq \kappa \exp \left(-n \delta+j_{1} \delta+\left(j_{2}+1\right) \delta / 2\right)
\end{aligned}
$$

Here we have $j_{2}>N(\delta, \xi)$ automatically, provided $c_{1}=c_{2}$ and $n_{1}$ large enough, since otherwise this critical point is periodic attracting hence not in $J(f)$.

Denote $n_{2}=n_{1}-j_{2}-1$ and continue, choosing $j_{3}, j_{4}, \ldots$, until an essential critical time $j_{k}$ does not exist; then the last pull-back is just the pull-back of $B_{k-1} \ni f^{j_{k}}(y)$ for $f^{j_{k}}$, containing $y, j_{k} \geq 0$. By this 'telescoping' construction and isolating annuli of moduli $\log ($ Const $/ \kappa)$, all the pull-backs $W_{s}$ of $B_{0}, s=1, \ldots, n$ have diameters not exceeding $\varepsilon$.

If there is more than one critical point in $J(f)$ then the proof should be modified in a standard way. It relies on the observation that for $n$ large enough the pull-backs under consideration have small diameters, so $j_{s}$ is small only if $f^{j_{s}+1}\left(c_{s}\right)=c_{s-1}$ which can happen consecutively only \# $(\operatorname{Crit}(f) \cap J(f))$ number of times, otherwise a critical point in $J(f)$ is periodic.

From Proposition 4.1 and Theorem 3.2 it follows 
Theorem 4.2 For every rational mapping $f: \widehat{\mathbb{C}} \rightarrow \widehat{\mathbb{C}}$ of degree at least 2 such that for every critical point $c \in J(f)$ the lower Lyapunov exponent $\chi(f(c))$ is non-negative, and for every $t>0$, the equality $P_{\text {spanning }}(t)=P_{\text {tree }}(t)$ holds.

Now let us invoke the following part of [9, Theorem 5.1]

Theorem 4.3 For every rational mapping $f: \widehat{\mathbb{C}} \rightarrow \widehat{\mathbb{C}}$ of degree at least 2 , such that there is exactly one critical point $c$ whose forward orbit has an accumulation point in $J(f)$ (i.e. $c \in J(f)$ or the forward trajectory of $c$ being attracted to a parabolic periodic orbit), we have $\underline{\chi}(f(c)) \geq 0$.

This and Theorem 4.2 yield

Corollary 4.4 Let $f: \widehat{\mathbb{C}} \rightarrow \widehat{\mathbb{C}}$ be a rational mapping of the Riemann sphere of degree at least 2, such that there is at most one critical point whose forward trajectory has an accumulation point in $J(f)$, then $P_{\text {spanning }}(t)=P_{\text {tree }}(t)$.

Without the assumption of weak backward stability, i.e. in the full generality, we can prove only the following in place of Theorem 3.2

Theorem 4.5 For every rational mapping $f: \widehat{\mathbb{C}} \rightarrow \widehat{\mathbb{C}}$ of degree at least 2 and for every $t>0$

$$
P_{\text {spanning }}(t)<\infty
$$

Proof We proceed as in the proof of Theorem 3.2 Part I, with small modifications. Notice that there exists $\Delta>0$ such that for an arbitrary $\varepsilon>0$ we have for $n$ large enough for every $x \in J(f)$ and every pull-back $V$ of $B(x, \exp (-n \Delta / 2))$ for $f^{j}, j=0, \ldots, n$, diam $V<\varepsilon$. This fact follows immediately from [3, Lemma 3.4].

Denote $\mathscr{B}:=\bigcup_{c \in \operatorname{Crit}(f) \cap J(f)} \bigcup_{j=1, \ldots, n} B(c, j)$, where $B(c, j):=B\left(f^{j}(c), r\right)$ where $r:=\exp (-n \Delta))$. Then we find $X \subset J(f) \backslash \mathscr{B}$ which is $r / 2$-spanning and $\# X \leq$ Const $\exp 2 n \Delta$. Then we find an $(\varepsilon, n)$-spanning set $Y$ as in the proof of Theorem 3.2 Part I. Finally, in place of the inequality (3.2), we just estimate $\left|\left(f^{n}\right)^{\prime}(y)\right|^{-t}$ for $y \in Y$. For this aim we shall use the following, see [3, Lemma 2.3]

Theorem 4.6 (Denker, Przytycki, Urbański) For every rational mapping $f: \widehat{\mathbb{C}} \rightarrow \widehat{\mathbb{C}}$ of degree at least 2 there exists $C_{f}>0$, which depends only on $f$ such that for every $z \in J(f)$

$$
\sum_{k=0}^{n-1}{ }^{\prime} \varphi(k) \leq C_{f} n, \quad n=1,2, \ldots
$$

where $\varphi(k)=-\log \rho\left(f^{k}(z)\right.$, Crit $\left.(f)\right)$ for the spherical metric $\rho$ and $\sum^{\prime}$ denotes the summation over all but at most $M=\#$ Crit indices.

Continuation of Proof of Theorem 4.5 We can now write, using (4.5),

$$
\left|\left(f^{n}\right)^{\prime}(y)\right|^{-t} \leq \exp (t C n) \exp (t C \Delta M n) L^{t M n} .
$$


for a constant $C \geq 0$ depending on $C_{f}$ and the multiplicities of the critical points. The factor $\exp (t C \Delta \bar{M} n) L^{t M n}$ takes care of (at most) $M$ integers $k$ omitted in $\sum^{\prime}$. For these $k$ we use $\rho\left(f^{k}(y), \operatorname{Crit}(f)\right) \geq L^{-(n-k)} \exp (-n \Delta)$, where $L=\sup \left|f^{\prime}\right|$, true since otherwise $\rho\left(f^{n}(y), f^{n-k}(\operatorname{Crit}(f))\right)<\exp -n \Delta$ which contradicts the definition of $r$ in $\mathscr{B}$ above.

Hence, collecting our estimates,

$$
P_{\text {spanning }}(t) \leq \log \operatorname{deg}(f)+t C+t C M \Delta+t M \log L
$$

\section{Geometric pressure via spanning sets: the real case}

We start from a notion refining the definition of safe, see Definition 2.1

Definition 5.1 For $(f, K) \in \mathscr{A}$ a point $z \in K$ is called safe from outer folds if for every $\eta>0$ and all $n \geq n(\eta)$ large enough, for every pull-back $W_{n}$ of $W=B(z, \exp (-\eta n))$ for $f^{n}$, intersecting $K$, there is a point $z_{n} \in \partial W_{n}$ such that $f^{j}\left(z_{n}\right) \in \widehat{I}$ for all $j=0,1, \ldots, n$.

Theorem 5.2 For every $(f, K) \in \mathscr{A}_{+}^{\mathrm{BD}}$, or $\mathscr{A}_{+}^{3}$, with all periodic orbits in $K$ hyperbolic repelling, weakly isolated, for every $t>0$ and every safe $z \in K$, it holds that $P_{\text {spanning }}(t) \geq P_{\text {tree }}(z, t)$.

If every periodic $z \in \partial \widehat{I}$ is safe from outer folds, then the equality of the pressures holds. In particular it holds provided $K=\widehat{I}=I$, namely it is a single interval

Proof $\mathbf{I}$. The CONSTRUCTION inequality: $P_{\text {spanning }}(t) \leq P_{\text {tree }}(t)$.

We mostly repeat parts of the proof of Theorem 2.6.

Fix an arbitrary safe $z \in K$ and $\delta$ adjusted to $\varepsilon$ as in the Definition of backward Lyapunov stability. Moreover assume $\delta<\delta_{0}$ as at the beginning of Proof of Lemma 2.10 .

For an arbitrary $0<\delta^{\prime} \leq \delta$ let $N=N\left(\delta^{\prime}\right)$ be such that $A=A\left(z, \delta^{\prime}\right):=\bigcup_{j=0, \ldots, N} f^{-j}(z) \cap K$ is $\delta^{\prime}$ dense in $K$.

We shall prove that the set $f^{-n}(A) \cap K$ itself happens to be an $(\varepsilon, n)$-spanning set, at least for a large subset of $K$, though to 'approximate' the remaining part of $K$, see two cases below, some additional points must be added to the spanning set.

Indeed, if for $w \in K, w^{\prime}=f^{n}(w) \in W=\left[z_{0}, z_{0}^{\prime}\right]$ with its endpoints belonging to $A$ whose distance is at most $\delta$ then for its pull-back $W_{n}=\left[z_{n}, z_{n}^{\prime}\right]$ containing $w$ we have for all $j=0, \ldots, n,\left|f^{j}\left(z_{n}\right)-f^{j}(w)\right|<\varepsilon$ (and the same for $z_{n}^{\prime}$ ). $z_{n}$ or $z_{n}^{\prime}$ belongs to $K$ by Lemma 2.11 .

A trouble is with $w$ such that $w^{\prime}=f^{n}(w)$ is not in any $W$ as above. Then, as in Proof of Theorem 2.6 there is a large gap (a component in $\mathbb{R} \backslash K$ ) of length at least $\delta / 4$ within the distance at most $\delta^{\prime}$ of $w^{\prime}$.

Then we have two cases.

(i) For some $m$ bounded by a constant depending only on $(f, K)$ and $\delta$, the point $f^{m}\left(w^{\prime}\right)$ belongs to some $W$ with endpoints $z_{0}, z_{0}^{\prime} \in A\left(z, \delta^{\prime}\right)$ for $\delta^{\prime}$ satisfying 
(2.10). Then $\rho_{n}\left(w, z_{n+m}\right) \leq \rho_{n+m}\left(w, z_{n+m}\right)<\varepsilon$ for an appropriate $z_{n+m}$ in the boundary of the pull-back of $W$ for $f^{n+m}$ containing $w$.

(ii) ${ }^{6}$ For some $n+m$ the point $w^{\prime \prime}=f^{n+m}(w)$ is close to a periodic point $p$ in the boundary of a large gap $G$.

Notice that in fact $p \in \partial \widehat{I}$, see [16, Lemma 2.9, Case 2]. Indeed, if $p$ and all other points of its periodic orbit belong to the interior of $\widehat{I}$, then also

$$
\bigcup f^{j}(G) \subset \operatorname{int} \widehat{I}
$$

Otherwise, if $j_{0}$ is the least integer such that $f^{j_{0}}(G)$ intersects $\partial \widehat{I}$ at a point $y$, then $y_{j_{0}}=\left(\left.f^{j_{0}}\right|_{G}\right)^{-1}(y) \in G$ and it belongs to $K$ since $y \in K$ and all $f^{j}\left(y_{j_{0}}\right), j=$ $1,2, \ldots$ belong to $\widehat{I}$ hence to $K$ by the maximality of $K$. This contradicts $G \cap K=\emptyset$. Thus (5.1) holds.

So all $f^{j}(G)$ are in $\widehat{I}$, hence, by the maximality, $G \subset K$. This again contradicts $G \cap K=\emptyset$.

Then, as at the end of Proof of Theorem 2.6, consider $\widehat{z} \in f^{-[\kappa n]}(z)$ belonging to $B=B(p, \exp -\eta n) \cap K$, for $\eta<\kappa \chi(p)$ where $\chi(p)$ is Lyapunov exponent at $p$. In particular $|p-\widehat{z}|<\exp -\eta n$. Denote $r=|p-\widehat{z}|$ and $B^{\prime}:=B(p, r) \subset B$.

If $w^{\prime \prime} \notin B^{\prime}$, then for some $k \leq \kappa \chi n$ the point $v=f^{k}\left(w^{\prime \prime}\right)$ is far from the periodic orbit of $p$ but $f^{k}$ is still invertible on $B\left(p,\left|p-w^{\prime \prime}\right|\right)$. In particular there exist $z_{0}, z_{0}^{\prime} \in A$ such that $\left|z_{0}-z_{0}^{\prime}\right|<\delta$ and $v \in\left[z_{0}, z_{0}^{\prime}\right]$. Hence $w^{\prime \prime} \in\left[z_{k}, z_{k}^{\prime}\right]$, the pull-back. Hence, as before, $w \in\left[z_{n+m+k}, z_{n+m+k}^{\prime}\right]$ where one of the ends say $z_{n+m+k}$ is in $K$ and $\rho_{n}\left(w, z_{n+m+k}\right)<\varepsilon$.

If $w^{\prime \prime} \in B^{\prime}$, then by the assumption that $p$ is safe from the outer fold for the constant $\eta$ for $n$ large enough, for $\left[z(w), z^{\prime}(w)\right]$ being the pull-back of $B$ for $f^{n+m}$ containing $w$, all $f^{j}(z(w)), j=0, \ldots, n+m$ belong to $\widehat{I}$ (or the same for $z^{\prime}(w)$ ). In particular $u:=f^{n+m}(z(w))$ is the point of $\partial B$ in $\widehat{I}$.

By our definitions, $\widehat{z}$ is between $w^{\prime \prime}$ and $u$. Since $w \in K, f^{j}(w) \in \widehat{I}$ for all $j \geq 0$. Hence there exists $\widehat{z}_{n+m} \in[w, z(w)] \cap f^{-n-m}(\widehat{z})$. such that $f^{j}\left(\widehat{z}_{n+m}\right) \in \widehat{I}$ as belonging to $f^{j}([w, z(w)]$ being intervals shorter than $\varepsilon$ with ends in $\widehat{I}$. These ends may be of the form $f^{j}(w), f^{j}(z(w))$ or $f^{i}(c)$ for a turning critical point $c \in K$ hence in $K \subset \widehat{I}$.

Hence $\widehat{z}_{n+m} \in K$ and $\rho_{n}\left(w, \widehat{z}_{n+m}\right) \leq \varepsilon$.

So, given $\varepsilon>0$ and safe $z \in K$, for all $\kappa>0$, for all $n$ large enough, the set

$$
\mathrm{SP}(z, n):=\bigcup_{0 \leq j \leq \kappa n+\operatorname{Const}(\varepsilon)} f^{-n-j}(\{z\})
$$

is $(n, \varepsilon)$-spanning. Const $(\varepsilon)$ depends on $N$ and $m$ above which depend on $\varepsilon$.

\footnotetext{
6 This case is harder than in Proof of Theorem 2.6, where the points $z, w$ were given a priori and we could choose $\delta^{\prime}$ appropriately.
} 
Next use $\left|\left(f^{j}\right)^{\prime}\right| \leq L^{j}$. We have, denoting $\widehat{n}=\kappa n+\operatorname{Const}(\varepsilon)$, for $\xi>0$,

$$
\begin{aligned}
& \sum_{x \in \operatorname{SP}(z, n)}\left|\left(f^{n}\right)^{\prime}(x)\right|^{-t} \leq \sum_{0 \leq j \leq \widehat{n}} Q_{n+j}(z, t) L^{j} \\
& \leq L^{\widehat{n}} \sum_{0 \leq j \leq \widehat{n}} \exp \left((n+j)\left(P_{\text {tree }}(z)+\xi\right)\right) .
\end{aligned}
$$

For $n \rightarrow \infty$ and $\kappa \rightarrow 0$ this holds for $\xi$ arbitrarily small and finally $P_{\text {spanning }}(t) \leq$ $P_{\text {tree }}(z, t)$.

Notice that unlike in Proof of Theorem 2.6 we have not needed here to compare the derivatives $\left|\left(f^{n}\right)^{\prime}(w)\right|$ and the shadowing $\left|\left(f^{n}\right)^{\prime}\left(z_{n}\right)\right|$. In particular we consider all $w$, rather than having $f^{n}(w)$ safe.

Notice finally that if $K=\widehat{I}=I$ is a single interval, then every $z \in \widehat{I}$ is safe from outer folds. Otherwise both ends $z_{n}, z_{n}^{\prime}$ of $W_{n}$ are outside $\widehat{I}$, since if, say, $z_{n} \in \widehat{I}$ then all $f^{j}\left(z_{n}\right) \in \widehat{I}$ by the forward invariance of $K=\widehat{I}$ here. So $z_{n}$ and $z_{n}^{\prime}$ are on the different sides of $I$. This is not possible since $W_{n}$ is short by backward Lyapunov stability of $f$.

II. The ALL inequality. The proof is the same as in the complex case, via $P_{\text {spanning }}(t) \geq P_{\text {hyp }}(t)$.

Example 5.3 We show that the assumption on the safety from outer folds is really needed in Theorem 5.2 above. We find examples of $(g, K, \widehat{I}, \mathbf{U}) \in \mathscr{A}_{+}^{\mathrm{BD}}$, weakly isolated, with all periodic orbits in $K$ hyperbolic repelling, for which $P_{\text {spanning }}(t)=\infty$ for all $t>0$.

- Consider quadratic polynomials $f_{a}(x)=a x(1-x)$ for $0<a<4$ large enough that the entropy of $f_{a}$ is positive. For each $a$ let $p_{a}$ denote the unique fixed point in the open interval $(0,1)$. It is repelling; let us make a small perturbation of $f_{a}$ close to $p_{a}$ so that $p_{a}$ becomes attracting and a repelling orbit $Q_{a}$ of period 2, being the boundary of $B_{0}(p) \subset(1 / 2,1)$ which is the immediate basin of attraction to $p_{a}$, is created.

One can do it in such a way that Schwarzian derivative $S g$ of the new map $g=g_{a}$ is negative except in $B_{0}(p)$. Write $Q_{a}=\left\{q_{a}, q_{a}^{\prime}\right\}$ with $q_{a}<q_{a}^{\prime}$. Omit the subscript a. Define

$$
g(x)= \begin{cases}f(x)-b \times(x-q)^{3}, & \text { if } q<x<p-\varepsilon \\ f(x)+b \times\left(q^{\prime}-x\right)^{3}, & \text { if } p+\varepsilon<x<q^{\prime} \\ p & \text { if } p-\varepsilon \leq x \leq p+\varepsilon \\ f(x), & \text { otherwise. }\end{cases}
$$

One can choose $\varepsilon>0$ arbitrarily small and $b>0$ so that the above function is continuous. Then $b$ is also small hence by $S f<0$ Schwarzian of $g$ stays negative except in $[p-\varepsilon, p+\varepsilon]$.

- Let $I_{a}=\left(I_{n}\right)_{n=1,2, \ldots, N}$ denote the kneading sequence for $g_{a}$, that is the sequence of letters $L, R, C$ depending whether $c_{n}=g_{a}^{n}(1 / 2)$ lies to the left of the critical 
point $1 / 2$, to the right of $1 / 2$, or at $1 / 2$. We put $N$ the least integer $n$ for which $I_{n}=1 / 2$. If no such integer exists we put $N=\infty$. See [2] for these definitions.

Let

$$
\underline{I}=R L R^{n_{1}} L R^{n_{2}} L R^{n_{3}} L \ldots,
$$

where $N=\infty$, all $n_{j}$ are finite positive, even, their sequence is increasing and $n_{j} \rightarrow \infty$ exponentially fast as $j \rightarrow \infty$.

$\underline{I}$ is a maximal sequence for every sequence $\left(n_{j}\right)$ satisfying above conditions, hence there exists $a$ such that $g=g_{a}$ has this kneading sequence, see [2, Theorem III.1.1].

For $I$ as above for $c_{n}$ left of $1 / 2$ we have $c_{n+1}$ right of $1 / 2$ and close to $q_{a}$, left of it (remember $Q_{a}=\left\{q_{a}, q_{a}^{\prime}\right\}$ with $q_{a}<q_{a}^{\prime}$ ). Next the trajectory $c_{n+2}, c_{n+3}, \ldots$ follows $Q_{a}$ outside of $\left[q_{a}, q_{a}^{\prime}\right]$, in the interval $(1 / 2,1)$ until $c_{n+n_{k}+1}$ occurs to the left of $1 / 2$ moreover to the left and close to the point being in the $g_{a}$-preimage of $q_{a}$ to the left of $1 / 2$.

- Now consider $\widehat{I}=\left[c_{2}, q_{a}\right] \cup\left[q_{a}^{\prime}, c_{1}\right], g$ restricted to a neighbourhood $\mathbf{U}$ of $\widehat{I}$ and $K$ the maximal forward invariant subset of $\widehat{I}$. Clearly $1 / 2 \in K$ since otherwise $g^{n}(1 / 2) \rightarrow p$ so $\underline{I}$ would consist solely of $R$ 's for $n$ large enough. $K=\widehat{I} \backslash B\left(p_{a}\right)$, where $B\left(p_{a}\right)$ is the basin of attraction by $g$ to $p_{a}$. Due to $S g<0$ on a neighbourhood $\mathbf{U}$ of $K$ we obtain $(g, K, \widehat{I}, \mathbf{U}) \in \mathscr{A}^{\mathrm{BD}}$, provided we prove

- Claim: $g$ is topologically transitive on $K$.

Let $a^{\prime}$ be so that the kneading sequence for $f=f_{a^{\prime}}$ is the same as for $g_{a}$, that is $\underline{I}$. Due to the lack of attracting and neutral periodic orbits for $f_{a^{\prime}}$ (otherwise $\underline{I}$ would be eventually periodic), there is a monotone increasing continuous semiconjugacy $h:\left[c_{2}, c_{1}\right] \rightarrow\left[c_{2}, c_{1}\right]$ such that $f \circ h=h \circ g . h$ is defined first in a standard way on $\mathscr{T}(g):=\bigcup_{n \geq 0} g^{-n}(1 / 2)$ to the corresponding $\mathscr{T}(f):=\bigcup_{n \geq 0} f^{-n}(1 / 2)$, increasing since the orders in the interval $\left[c_{2}, c_{1}\right]$ of points in these sets are (combinatorially) the same, due to the same kneading sequences.

The mapping $h$ can be continuously extended to the closures, and notice that cl $\mathscr{T}(f)=\left[c_{2}, c_{1}\right]$ due to the absence of wandering intervals for $f$.

This $h$ collapses $B_{0}(p)$ and its $g^{n}$-preimages to points, provided we extend $h$ to these gaps by constant functions. In other words $h$ identifies the pairs of points being ends of gaps $B(p)$ being components in the basin of $p$. There are no other gaps in $\left[c_{2}, c_{1}\right] \backslash \mathrm{cl} \mathscr{T}(g)$ since there are no wandering intervals (see [10]) and no attracting or neutral periodic orbits other than $p$. This in turn holds since the Schwarzian $S g$ is negative outside $B(p)$ so the basin of such an orbit would contain a critical point that is $1 / 2$ which is not possible since $\underline{I}$ is not eventually periodic. Therefore $h$ is injective on $K$ except the abovementioned pairs of points.

Notice that our $I$ is not a *-product, see [2, Section II.2] for the definition. Hence there is no interval $T \subset I_{f}=\left[f^{2}(1 / 2), f(1 / 2)\right]$ such that $f^{k}(T) \subset T$ for some $k>1$ containing $1 / 2$ with $f^{k}$ unimodal on it (i.e. with one turning point), i.e. there is no renormalization interval. (In other words $f$ is not renormalizable). This follows from [2, Corollary II.7.14].

Consider now any interval $T \subset I_{f}$ and $V=\bigcup_{j \geq 0} f^{j}(T)$. By definition $V$ is forward invariant. Let $W$ be a connected component of $V$. Then there are integers $0 \leq k_{1}<k_{2}$ such that $f^{k_{1}}(W) \cap f^{k_{2}}(W) \neq \emptyset$ since $W$ is non-wandering, see [10, 
Chapter IV, Theorem A] for the non-existence of wandering intervals. Hence, for $k=k_{2}-k_{1}$, and $W^{\prime}=f^{k_{1}}(W), f^{k}\left(W^{\prime}\right) \subset W^{\prime}$. We consider $k$ the smallest such integer. We can assume that $1 / 2 \in W^{\prime}$ (or some $f^{j}\left(W^{\prime}\right)$ ), since otherwise $W$ would be attracted to a periodic orbit and we have assumed such orbits do not exist. No $f^{\ell}\left(W^{\prime}\right), 0<\ell<k$ contains $1 / 2$ by its disjointness from $W^{\prime}$. So $f^{k}$ is unimodal on $W^{\prime}$. So $k=1$, since otherwise $f$ would be renormalizable. So $f(1 / 2)$ and $f^{2}(1 / 2)$, the end points of $I_{f}$, belong to $W^{\prime}$. Hence $V=I_{f}$, hence $f$ is topologically transitive on $I_{f}$.

This due to our semiconjugacy and the fact that $K$ has no isolated points, implies the topological transitivity of $g$ on $K$. The Claim is proved.

The property we proved in particular, that for every open $W \subset K$ there exists $k$ such that $\left(\left.g\right|_{K}\right)^{k}(W)=K$, is called topological exactness or leo - "locally eventually onto". This is stronger than topological transitivity. See [16, Lemma A7] for a discussion of a general case.

Since the topological entropy of $\left.g\right|_{K}$ is positive we can write $(g, K, \widehat{I}, \mathbf{U}) \in \mathscr{A}_{+}^{\mathrm{BD}}$.

- Notice that $K$ is weakly isolated for $g$ on $\mathbf{U}$, see Definition 2.5. This is so because if a periodic trajectory $P$ in $\mathbf{U}$ has a point $z \notin K$ then $z$ belongs to the basin of attraction to $p$, i.e. $g^{n}(z) \rightarrow p$. In other words the trajectory $\left.g\right|_{\mathbf{U}} ^{n}(z)$ leaves $\mathbf{U}$. Hence $P \subset K$. Note that above argument proves the weak isolation property in general situations, namely if $K$ is Julia set in the sense of [10, Chapter IV, Lemma] i.e. the domain being an interval with the basins of attracting or neutral periodic orbits removed (provided there is a finite number of them).

- Notice that $q_{a}$ is not safe from outer folds, see Definition 5.1. Indeed. Denote $2+\sum_{j=1, \ldots k} n_{j}+k+1$ by $m_{k}$. The summands $n_{j}$ correspond to the blocks of $R$ 's, the first summand 2 corresponds to the starting $R L$ and the final 1 to the first $R$ in the $k+1$ 'th block of $R$ 's. We obtain $\left|c_{m_{k}}-q_{a}\right| \leq$ Const $(a) \exp \left(-n_{k+1} \chi\left(q_{a}\right)\right)$, where $\chi\left(q_{a}\right)=\frac{1}{2} \log \left|\left(g^{2}\right)^{\prime}\left(q_{a}\right)\right|$. Consider the pullback $W_{m_{k}}$ of $W=B\left(q_{a}, \exp \left(-\eta m_{k}\right)\right)$ for $g^{m_{k}}$ containing $\frac{1}{2}$.

The critical point $1 / 2$ is not recurrent since $c_{m_{k}-1}$ corresponding to $L$ approach to the point symmetric to $q_{a}$ since $n_{j}$ grow, so they are in the distance from $c_{2}$ bounded away from 0 . Hence the only points we need to care about, $c_{m_{k}-3}$ are in the distance from $1 / 2$ also bounded away from 0 .

Hence for all $n=1,2, \ldots, m_{k}-1$ the map $g$ is injective on $g^{n}\left(W_{m_{k}}\right)$ but $g$ has a turning critical point $1 / 2$ in $W_{m_{k}}$. Using the assumption that all $n_{j}$ are even we conclude that each $g^{m_{k}}$ has a minimum at $1 / 2$, hence if $n_{k+1} \chi\left(q_{a}\right) \gg m_{k} \eta$ the boundary points of $W_{m_{k}}$ are mapped by $g^{m_{k}}$ into the gap (basin $B_{0}(p)$ ). In other words $\partial W_{m_{k}} \subset B_{m_{k}} \cup B_{m_{k}}^{\prime}$ the latter being the union of a symmetric pair of pull-backs of $B_{0}(p)$ for $g^{m_{k}}$ on both sides of $1 / 2$ (Fig. 1).

- Imposing sufficient growth of $n_{j}$, e.g.

$$
n_{j+1} / n_{j} \rightarrow \infty \text {, }
$$

we get a counterexample to $P_{\text {spanning }}(t) \leq P_{\text {tree }}(z, t)$. Indeed, consider an arbitrary $x \in K$ which $(n, \varepsilon)$-close to $1 / 2$ for $n=m_{k}$. 


\section{F. PRZYTYCKI}

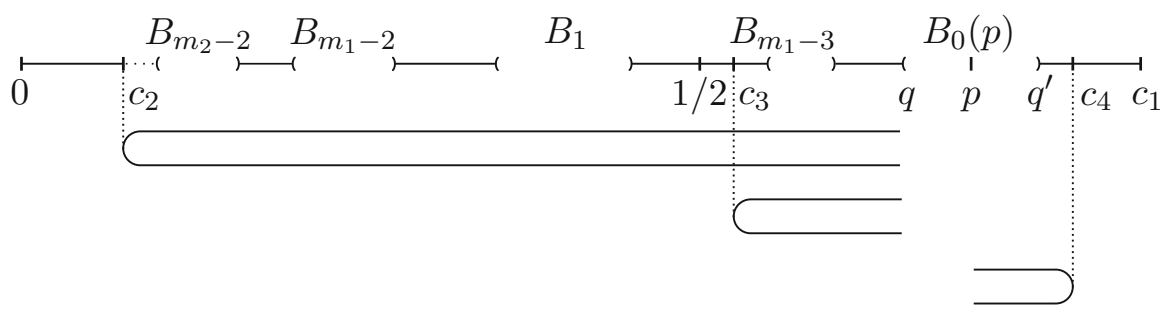

Fig. 1 Pull-backs of $B_{0}(p)$

Due to the non-recurrence of $1 / 2$, see above, $g$ is expanding on the limit set $\omega(1 / 2)$, see Definition 2.3 and e.g. Mañé's theorem: [10, Section III.5 Corollary 1]. Denote the expanding constant by $\lambda$, compare Definition 2.3 .

Hence for $\varepsilon$ small enough and an integer $N$ such that all $g^{j}(1 / 2), j \geq N$ are close to $\omega(1 / 2)$, if $\left|g^{j}(1 / 2)-g^{j}(x)\right| \leq \varepsilon$ for all $j: N \leq j \leq n$, then for all $N \leq j \leq n$, $g^{j}(x) \in g^{j}\left(W_{n}\right)$, where $W_{n}$ is the pull-back of $W$ as above, but for $W=B\left(q_{a}, \varepsilon\right)$ (unlike above). Then this holds also automatically also for $0 \leq j<N$, maybe on the cost of taking a smaller $\varepsilon$.

Suppose $n_{j+1} \gg m_{j}$. Then diam $g^{n}\left(W_{n}\right) \cap \widehat{I} \leq \exp -C n$ for $C$ large. Hence for all $0<j \leq n,\left|g^{j}(x)-c_{j}\right| \leq \exp -C n$ and $|x-1 / 2| \leq \exp -C n / 2$. Hence $\left|\left(f^{n}\right)^{\prime}(x)\right| \leq$ $\lambda^{2 n} \exp -C n / 2$. Hence, for every $(n, \varepsilon)$-spanning set $Y \subset K, \sum_{y \in Y}\left|\left(g^{n}\right)^{\prime}(y)\right|^{-t} \geq$ $\lambda^{-t 2 n} \exp C n / 2$. The assumption (5.3) allows to have $C$ arbitrarily large.

We conclude that $P_{\text {spanning }}(t)=\infty$.

Remark 5.4 1. In the example above $K$ is not uniformly perfect (considered in the plane), unlike in the complex case where the uniformly perfect property of Julia set allowed us to prove Theorem 4.

2. In this example the so-called Bowen's periodic specification property does not hold. This property is defined for any continuous map $f: X \rightarrow X$ of a compact $X$ as follows: For every $\varepsilon>0$ there exists an integer $N$ such that for every $x \in X$ and every integer $n \geq 0$ there exists $y \in X$ of period $k: n \leq k \leq n+N$ such that for every $0 \leq j \leq n, \operatorname{dist}\left(f^{j}(x), f^{j}(y)\right) \leq \varepsilon$.

Even a weaker periodic specification does not hold, where $N=N(\varepsilon)$ is replaced by $N(n, \varepsilon)$ for $\varepsilon$ small enough (see the survey [8]). Namely for every function $N(n, \varepsilon)$ there exists $a$ such that for $g_{a}$ with an appropriate kneading sequence $\underline{I}$ the specification with $N(\varepsilon, n)$ does not hold. Consider blocks of the $g$-trajectories $1 / 2, c_{1}, c_{2}, \ldots c_{m_{j}}$ with $n_{k}$ growing fast enough. Then for every $y$ being $\left(m_{j}, \varepsilon\right)$ close to $1 / 2, y$ is in fact $\xi$-close to $1 / 2$ for $\xi>0$ arbitrarily small, depending on $n_{j+1}$. Then the period of $y$ must be long since otherwise $y$ would be an attracting periodic point.

3. One can have an additional insight in the topological dynamics of $g_{a}$ or $f=f_{a^{\prime}}$ if one uses the existence of a semiconjugacy of $f$ to a tent map $\tau$ (of slopes $\pm h_{\text {top }}(f$ ), see [11, Theorem 7.4], which must be a conjugacy since $f$ has no renormalization or wandering interval [10, Chapter IV, Theorem A]. 
Open Access This article is distributed under the terms of the Creative Commons Attribution 4.0 International License (http://creativecommons.org/licenses/by/4.0/), which permits unrestricted use, distribution, and reproduction in any medium, provided you give appropriate credit to the original author(s) and the source, provide a link to the Creative Commons license, and indicate if changes were made.

\section{References}

1. Bowen, R.: Equilibrium States and the Ergodic Theory of Anosov Diffeomorphisms, Lecture Notes in Mathematics, vol. 470. Springer, Berlin (1975)

2. Collet, P., Eckmann, J.-P.: Iterated Maps on the Interval as Dynamical Systems. Series Progress in Physics. Birhhäuser, Boston (1980)

3. Denker, M., Przytycki, F., Urbański, M.: On the transfer operator for rational functions on the Riemann sphere. Ergod. Theory Dyn. Syst. 16, 255-266 (1996)

4. Gelfert, K., Przytycki, F., Rams, M.: Lyapunov spectrum for multimodal maps. Ergod. Theory Dyn. Syst. 36.5, 1441-1493 (2016). arXiv:1406.4731v2

5. Graczyk, J., Smirnov, S.: Collet, Eckmann and Hölder. Invent. Math. 133, 69-96 (1998)

6. Hall, R.R., Hayman, W.K.: Hyperbolic distance and distinct zeros of the Riemann zeta-function in small regions. J. Reine Angew. Math. 526, 35-59 (2000)

7. Järvi, P., Vuorinen, M.: Uniformly perfect sets and quasiregular mappings. J. Lond. Math. Soc. 54, 515-529 (1996)

8. Kwietniak, D., Łącka, M., Oprocha, P.: A panorama of specification-like properties and their consequences. Dyn. Numbers. Contemp. Math. 669, 155-186 (2016). arXiv:1503.07355v2

9. Levin, G., Przytycki, F., Shen, W.: The Lyapunov exponent of holomorphic maps. Invent. Math. 205, 363-382 (2016). arXiv:1304.6178v2

10. de Melo, W., van Strien, S.: One-Dimensional Dynamics. Springer, Berlin (1994)

11. Milnor, J., Thurston, W.: On Iterated Maps of the Interval, Dynamical Systems, Lecture Notes in Mathematics 1342, pp. 465-563. Springer, Berlin (1988)

12. Przytycki, F.: Lyapunov characteristic exponents are nonnegative. Proc. Am. Math. Soc. 119, 309-317 (1993)

13. Przytycki, F.: Iteration of holomorphic Collet-Eckmann maps: conformal and invariant measures. Appendix: on non-renormalizable quadratic polynomials. Trans. Am. Math. Soc. 350, 717-742 (1998)

14. Przytycki, F.: Conical limit set and Poincaré exponent for iterations of rational functions. Trans. Am. Math. Soc. 351, 2081-2099 (1999)

15. Przytycki, F., Rivera-Letelier, J.: Nice inducing schemes and the thermodynamics of rational maps. Commun. Math. Phys. 301(3), 661-707 (2011)

16. Przytycki, F., Rivera-Letelier, J.: Geometric pressure for multimodal maps of the interval (to appear in Memoirs of the Amer. Math. Soc) arXiv:1405.2443

17. Przytycki, F., Rivera-Letelier, J., Smirnov, S.: Equivalence and topological invariance of conditions for non-uniform hyperbolicity in the iteration of rational maps. Invent. Math. 151, 29-63 (2003)

18. Przytycki, F., Rivera-Letelier, J., Smirnov, S.: Equality of pressures for rational functions. Ergod. Theory Dyn. Syst. 24, 891-914 (2004)

19. Przytycki, F., Urbański, M.: Conformal Fractals: Ergodic Theory Methods, London Mathematical Society Lecture Note Series, vol. 371. Cambridge University Press (2010)

20. Rivera-Letelier, J.: Asymptotic expansion of smooth interval maps. arXiv:1204.3071v2

21. Ruelle, D.: Thermodynamic Formalism, Encyclopedia of Mathematics and Its Applications, vol. 5. Addison-Wesley Publ. Co., London (1978)

22. Sinai, Y.G.: Theory of Phase Transitions: Rigorous Results. Akadémiai Kiadó, Budapest (1982)

23. Sugawa, T.: Uniformly perfect sets: analytic and geometric acpects. Sugaku Expos. 16(2), 225-242 (2003)

24. Walters, P.: An Introduction to Ergodic Theory. Springer, Berlin (1982) 